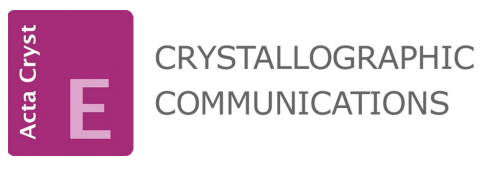

ISSN 2056-9890

Received 18 April 2019

Accepted 12 July 2019

Edited by L. Fabian, University of East Anglia, England

₹ Current address: Xellia Ltd, HR-10000 Zagreb, Croatia.

Keywords: crystal structure; memantine; X-ray diffraction; differential scanning calorimetry (DSC); thermogravimetric analysis (TGA); IR spectroscopy.

CCDC reference: 1942388

Supporting information: this article has supporting information at journals.iucr.org/e

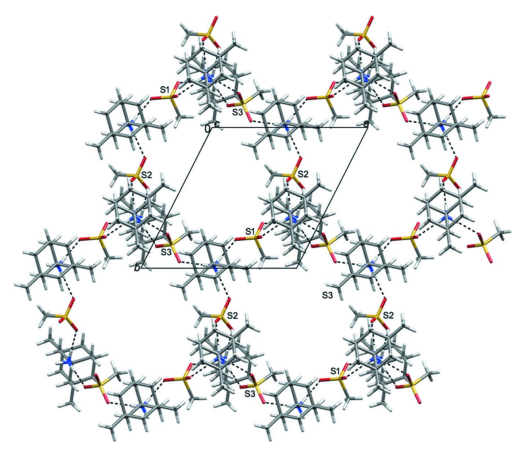

OPEN $\odot$ ACCESS

\section{(3,5-Dimethyladamantan-1-yl)ammonium methane- sulfonate (memantinium mesylate): synthesis, structure and solid-state properties}

\author{
Mihaela Tuksar, ${ }^{a}$ Mirta Rubčić ${ }^{b *}$ and Ernest Meštrovića ${ }^{a} \neq$ \\ aPLIVA Croatia Ltd., TAPI Research and Development, Prilaz baruna Filipovića 29, HR-10000 Zagreb, Croatia, and \\ ${ }^{\mathbf{b}}$ Department of Chemistry, Faculty of Science, University of Zagreb, Horvatovac 102a, HR-10000 Zagreb, Croatia. \\ *Correspondence e-mail: mirta@chem.pmf.hr
}

The asymmetric unit of the title compound, $\mathrm{C}_{12} \mathrm{H}_{22} \mathrm{~N}^{+} \cdot \mathrm{CH}_{3} \mathrm{O}_{3} \mathrm{~S}^{-}$, consists of three (3,5-dimethyladamantan-1-yl)ammonium cations, $\mathrm{C}_{12} \mathrm{H}_{22} \mathrm{~N}^{+}$, and three methanesulfonate anions, $\mathrm{CH}_{3} \mathrm{O}_{3} \mathrm{~S}^{-}$. In the crystal, the cations and anions associate via $\mathrm{N}-\mathrm{H} \cdots \mathrm{O}$ hydrogen bonds into layers, parallel to the (001) plane, which include large supramolecular hydrogen-bonded rings.

\section{Chemical context}

Memantine or 3,5-dimethyladamantane-1-ylamine is an active pharmaceutical ingredient which acts as an uncompetitive NMDA receptor antagonist (Reisberg et al., 2003; Rammes et al., 2008; Parsons et al., 2013). The compound was approved for the treatment of moderate-to-severe Alzheimer's disease and is currently marketed as the chloride salt. The crystal structure of memantinium chloride 0.1-hydrate has previously been described (Lou et al., 2009). Herein we report the structure of an alternative salt, (3,5-dimethyladamantan-1yl)ammonium methanesulfonate (I) (memantinium mesylate), developed with the aim of producing a material with physicochemical properties superior to those of memantinium chloride.

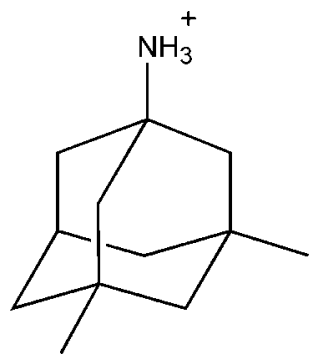<smiles>CS(=O)(=O)[O-]</smiles>

\section{Structural commentary}

The asymmetric unit of (3,5-dimethyladamantan-1-yl)ammonium methanesulfonate, (I) (Fig. 1) consists of three crystallographically independent (3,5-dimethyladamantan-1-yl)ammonium cations and three methanesulfonate anions. The structure of the cations is rigid, with all four six-membered rings of the adamantane core of the (3,5-dimethyladamantan1-yl)ammonium cations assuming a typical chair conformation. No significant geometrical differences are observed between the independent cations, or between the methane- 
Table 1

Hydrogen-bond geometry $\left(\AA,^{\circ}\right)$.

\begin{tabular}{lllll}
\hline$D-\mathrm{H} \cdots A$ & $D-\mathrm{H}$ & $\mathrm{H} \cdots A$ & $D \cdots A$ & $D-\mathrm{H} \cdots A$ \\
\hline $\mathrm{N} 1-\mathrm{H} 1 A \cdots \mathrm{O} 7^{\mathrm{i}}$ & $0.90(2)$ & $1.92(2)$ & $2.819(2)$ & $177(2)$ \\
$\mathrm{N} 1-\mathrm{H} 1 B \cdots \mathrm{O} 1$ & $0.90(2)$ & $1.94(2)$ & $2.833(2)$ & $179(2)$ \\
$\mathrm{N} 1-\mathrm{H} 1 C \cdots \mathrm{O} 4$ & $0.89(2)$ & $1.96(2)$ & $2.844(2)$ & $170(2)$ \\
$\mathrm{N} 2-\mathrm{H} 2 A \cdots \mathrm{O} 2^{\mathrm{ii}}$ & $0.88(2)$ & $1.92(2)$ & $2.7991(18)$ & $179(2)$ \\
$\mathrm{N} 2-\mathrm{H} 2 B \cdots \mathrm{O} 9^{\mathrm{ii}}$ & $0.87(2)$ & $1.94(2)$ & $2.8090(19)$ & $175(2)$ \\
$\mathrm{N} 2-\mathrm{H} 2 C \cdots \mathrm{O} 6$ & $0.90(2)$ & $1.90(2)$ & $2.7923(19)$ & $177(2)$ \\
$\mathrm{N} 3-\mathrm{H} 3 A \cdots \mathrm{O} 3$ & $0.90(2)$ & $1.91(2)$ & $2.7717(19)$ & $159(2)$ \\
$\mathrm{N} 3-\mathrm{H} 3 B \cdots \mathrm{O} 5$ & $0.90(2)$ & $1.89(2)$ & $2.7752(19)$ & $172(2)$ \\
$\mathrm{N} 3-\mathrm{H} 3 C \cdots \mathrm{O} 8^{\mathrm{i}}$ & $0.89(2)$ & $1.90(2)$ & $2.785(2)$ & $172(2)$ \\
$\mathrm{C} 39-\mathrm{H} 39 B \cdots \mathrm{O} 6$ & \\
& 0.96 & 2.59 & $3.423(3)$ & 145
\end{tabular}

Symmetry codes: (i) $x+1, y, z$; (ii) $x, y-1, z$; (iii) $-x+1,-y+1,-z+1$.

sulfonate anions. The (3,5-dimethyladamantan-1-yl)ammonium cations are achiral. They possess a plane of symmetry by which two enantiomorphic halves of the ion, containing chiral centers (C3 and C5, C15 and C17, C27 and C29), are reflections of each other.

\section{Supramolecular features}

The crystal packing of the title compound is characterized by hydrogen-bonding interactions between the protonated amino groups of cations and the oxygen atoms of the methanesulfonate anions (Table 1, Fig. 2). Each hydrogen atom of the protonated amino groups of the (3,5-dimethyladamantan-1yl)ammonium cations is engaged in hydrogen bonding with the neighbouring methanesulfonate anions. While each of the established $\mathrm{N}-\mathrm{H} \cdots \mathrm{O}$ hydrogen bonds has a characteristic $D_{1}^{1}(2)$ graph-set motif, they combine into larger $R_{4}^{4}(12)$ motifs (Fig. 2). Assemblies formed in such a way are supported by weaker $\mathrm{C}-\mathrm{H} \cdots \mathrm{O}$ contacts, as shown in Fig. 2. Such connec-

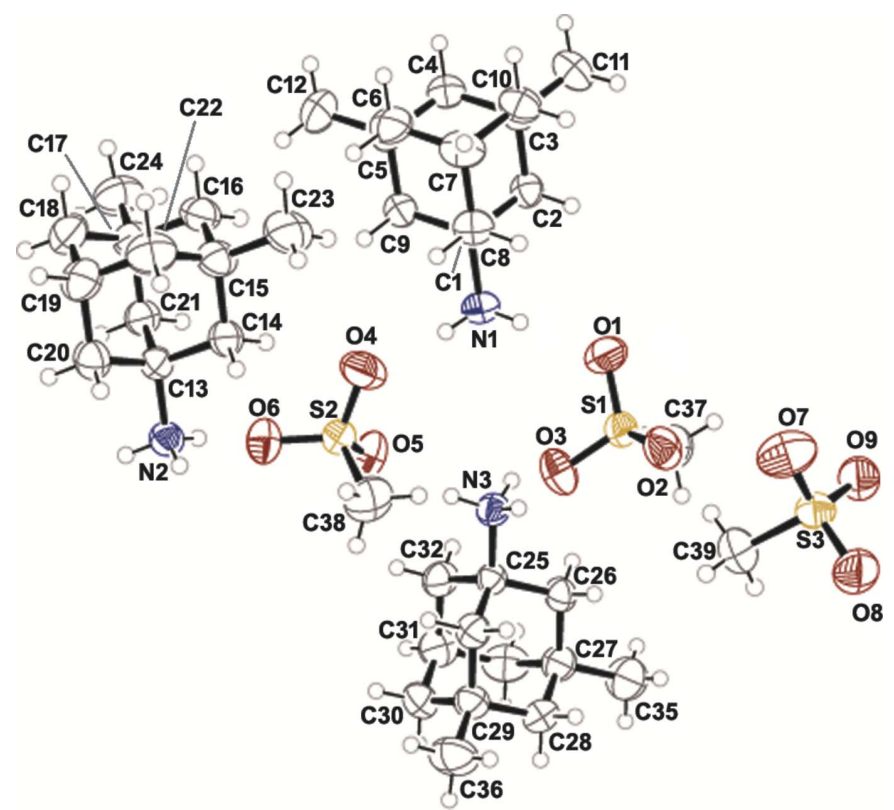

Figure 1

ORTEP plot of the title compound. Displacement ellipsoids are drawn at the $50 \%$ probability level and $\mathrm{H}$ atoms are shown as spheres of arbitrary small radii. tivity leads to the formation of supramolecular layers parallel to the (001) plane, which involve large hydrogen-bonded rings (Fig. 3).

\section{Database survey}

A search of the Cambridge Structural Database (CSD version 5.40, update of November 2018; Groom et al., 2016) for structures containing the (3,5-dimethyladamantan-1-yl)ammonium cation gave four hits: (3,5-dimethyl-1-adamantyl)ammonium chloride hydrate (DUCYAC; Lou et al., 2009), 3,5dimethyladamantane-1-ammonium cucurbit[8]uril chloride hexacosahydrate (GAWLIC, Hostaš et al., 2016), cucurbit[7] uril memantine clathrate chloride hydrate (SULZIJ, McInnes et al., 2010) and 3,5-dimethyladamantan-1-yl-

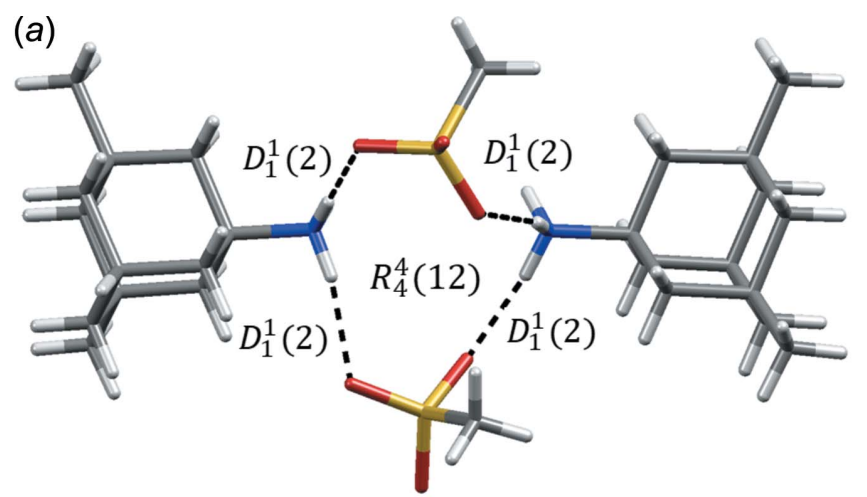

(b)

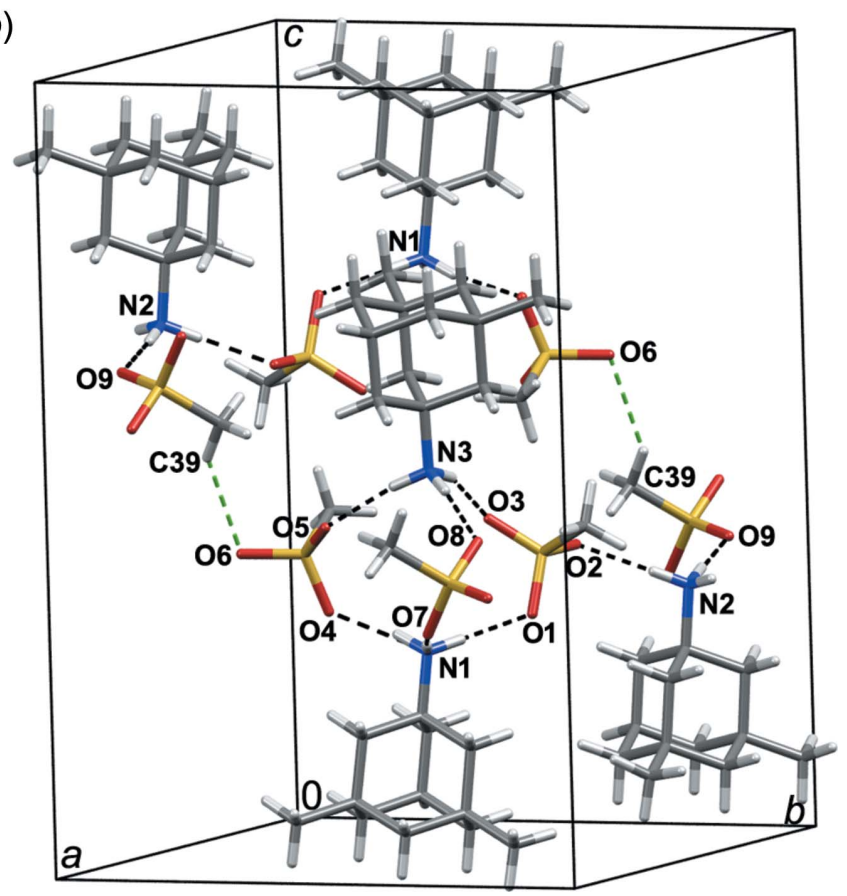

Figure 2

(a) A view of the $D_{1}{ }^{1}(2)$ and $R_{4}^{4}(12)$ motifs formed via $\mathrm{N}-\mathrm{H} \cdots \mathrm{O}$ hydrogen bonds. (b) Crystal packing of the title compound showing relevant hydrogen bonds and $\mathrm{C}-\mathrm{H} \cdots \mathrm{O}$ contacts. Hydrogen bonds are indicated by black dashed lines, while the $\mathrm{C}-\mathrm{H} \cdots \mathrm{O}$ contacts are shown as green dashed lines. 
(a)

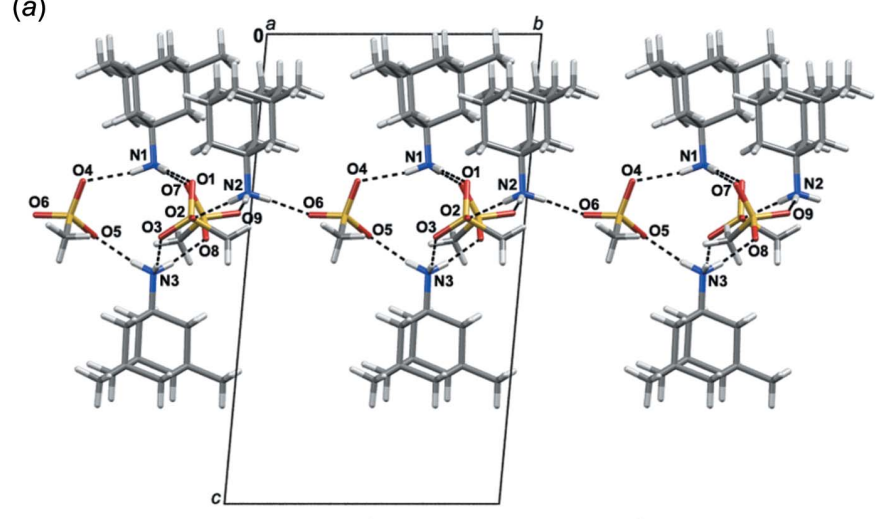

(b)

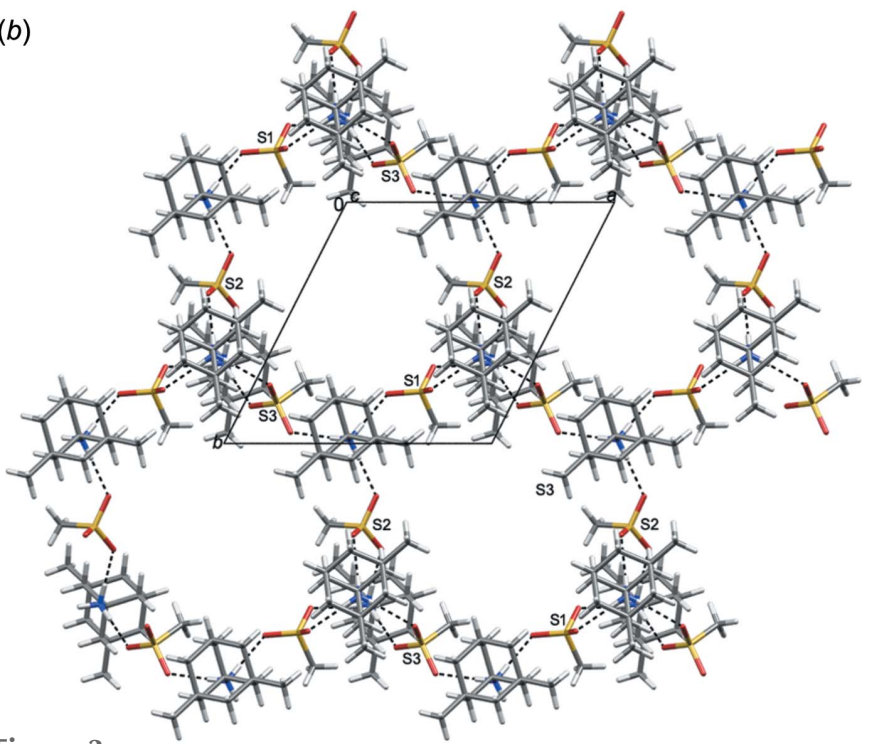

Figure 3

Crystal packing of the title compound showing the layers parallel to (001) based on hydrogen bonded rings. View of the structure: $(a)$ along the [100] direction; $(b)$ along the [001] direction. Hydrogen bonds are indicated by dashed lines.

ammonium 2,4,6-triisopropylbenzenesulfonate (YECDIW, Tkachev et al., 2017). Among these, the structure of 3,5-dimethyladamantan-1-ylammonium 2,4,6-triisopropylbenzenesulfonate shows the greatest similarity in its hydrogen-bonding motifs with those observed in the title compound. In the structure of YECDIW, $\mathrm{N}-\mathrm{H} \cdots \mathrm{O}$ hydrogen bonds having a $D_{1}^{1}(2)$ graph-set motif dominate the crystal packing. However, in contrast to the hydrogen-bonded layers in the title structure, a complex chain-like hydrogen-bonding network is formed. Such differences can be attributed, at least to some extent, to the distinct steric demands of the anions present in these structures.

\section{Hirshfeld surface analysis}

The Hirshfeld surfaces for the cations and anions constituting the asymmetric unit of (I) were calculated using CrystalExplorer17 (Turner et al., 2017) and are shown in Fig. 4. Mapping the $d_{\text {norm }}$ values on the corresponding Hirshfeld surface allows a detailed analysis of hydrogen bonds and short intermolecular contacts (Spackman \& Jayatilaka, 2009). In this
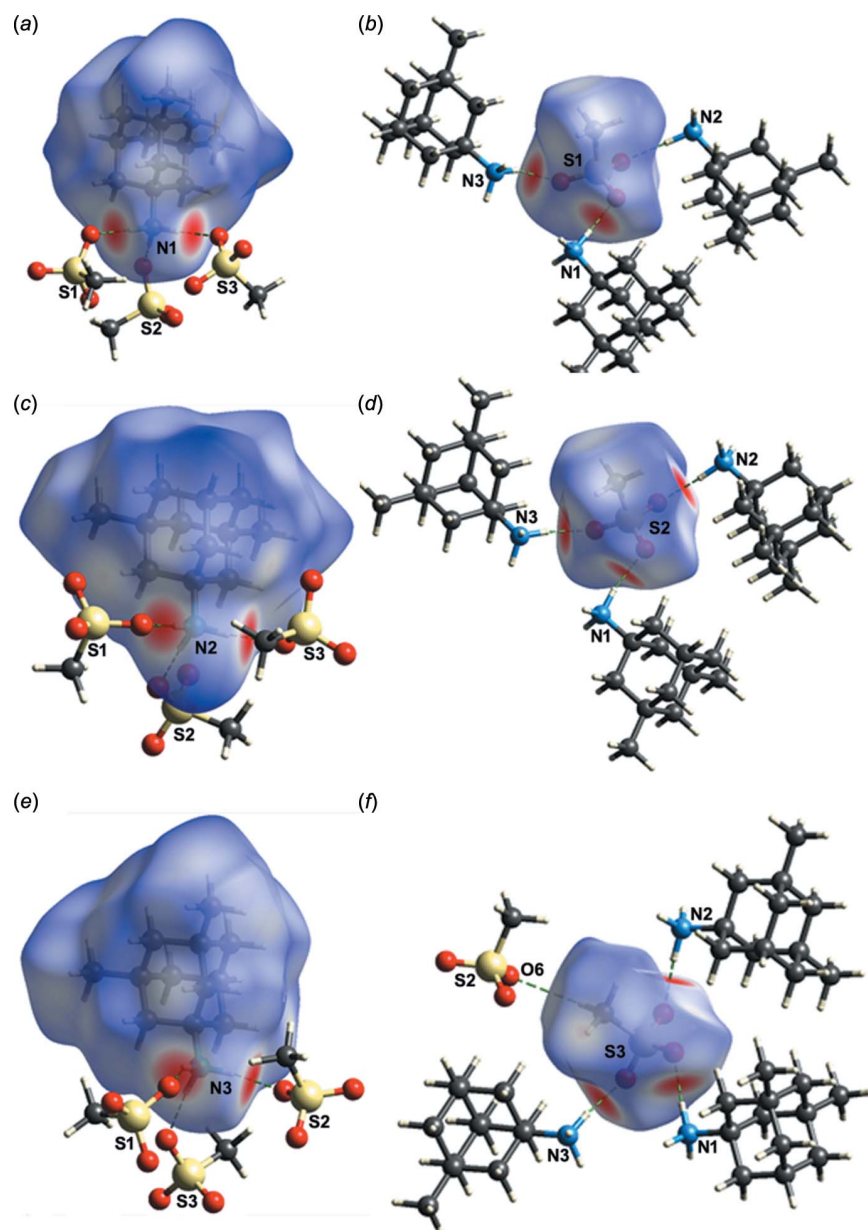

$(f)$

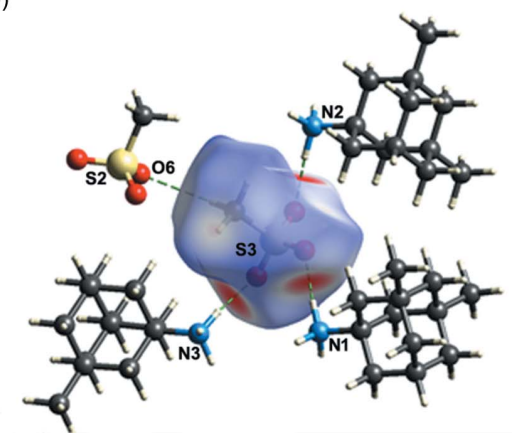

Figure 4

Views of the Hirshfeld surfaces mapped over $d_{\text {norm }}$ for: $(a)$ the N1containing cation; $(b)$ the S1-containing anion, $(c)$ the N2-containing cation; $(d)$ the S2-containing anion, $(e)$ the N3-containing cation and $(f)$ the S3-containing anion (range: -0.6178 to 1.7852 a.u.).

case, red spots indicate $\mathrm{N}-\mathrm{H} \cdots \mathrm{O}$ hydrogen bonds, blue regions correspond to positive $d_{\text {norm }}$ values, and white areas indicate contacts of equal length to the sum of the van der Waals radii, i.e. $d_{\text {norm }}$ is 0 . While the Hirshfeld surfaces for the three cations appear similar to each other, the two-dimensional fingerprint plots reveal distinctive differences between them. The full two-dimensional fingerprint plots along with the decomposed ones, displaying the contributions of the relevant contacts, are shown in Fig. 5. It can be seen that the $\mathrm{N} 3$-containing cation has the largest contribution of $\mathrm{H} \cdots \mathrm{O} /$ $\mathrm{O} \cdots \mathrm{H}$ contacts $(23.9 \%)$, while for the N1- and N2-containing cations this contribution amounts to 14.9 and $17.1 \%$, respectively. Analysis of the fingerprint plots for the anions reveals that they have fairly similar environments within the crystal and consequently a comparable distribution of the intermolecular contacts (Fig. 5).

\section{Synthesis and crystallization}

To a solution of $10.0 \mathrm{~g}$ of (3,5-dimethyladamantan-1-yl)ammonium chloride (supplied by PLIVA Croatia Ltd.) in $300 \mathrm{ml}$ 
(a)

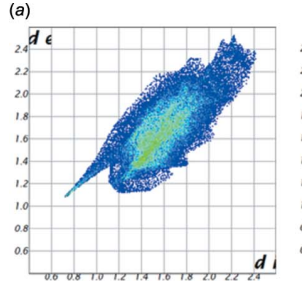

(b)

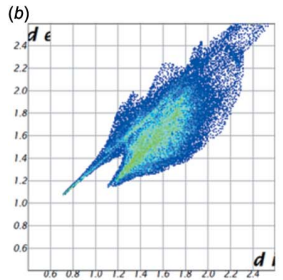

(c)

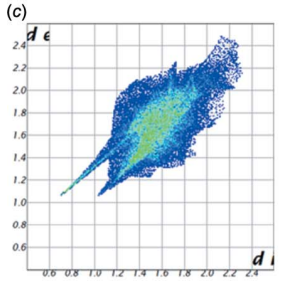

(d)

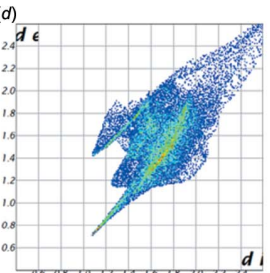

(e)
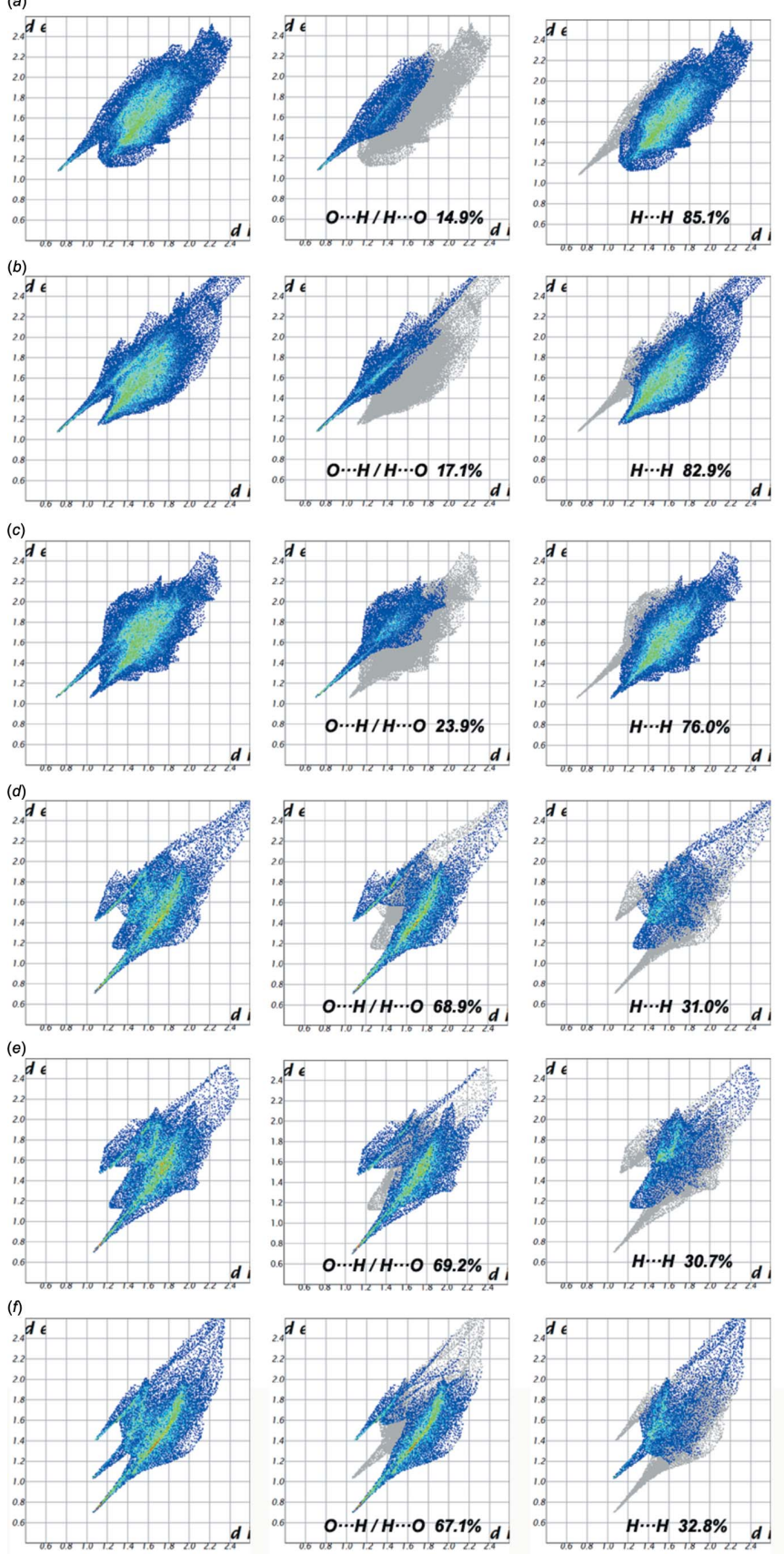

Figure 5

The fingerprint plots for the ions constituting the asymmetric unit of (I): (a) the N1-containing cation; $(b)$ the N2-containing cation, $(c)$ the N3containing cation; $(d)$ the S1-containing anion, $(e)$ the S2-containing anion and $(f)$ the S3-containing anion. Left side: full fingerprint plot, middle: contribution of the $\mathrm{H} \cdots \mathrm{O} / \mathrm{O} \cdots \mathrm{H}$ contacts, and right side: contribution of the $\mathrm{H} \cdots \mathrm{H}$ contacts to the intermolecular interactions.

of water, $140 \mathrm{ml}$ of toluene was added and the $\mathrm{pH}$ adjusted to about 10.7 by using $40 \% \mathrm{NaOH}(\mathrm{aq})$. The toluene and water layers were separated. To the toluene solution of 3,5-dimethyladamantane-1-ylamine, $3.3 \mathrm{ml}$ of methanesulfonic acid at $293-298 \mathrm{~K}$ was added. The reaction mixture was stirred at 293-298 K for $1 \mathrm{~h}$, cooled to $273-278 \mathrm{~K}$ and stirred at that temperature for $1 \mathrm{~h}$. The resulting crystals were filtered off, washed with toluene and dried at $313 \mathrm{~K} / 20 \mathrm{mbar}$ for about
$15 \mathrm{~h}$. The obtained solid was slurried in $125 \mathrm{ml}$ of acetone at 293-298 K for about $18 \mathrm{~h}$, filtered off, washed with acetone and dried at $313 \mathrm{~K} / 20 \mathrm{mbar}$ for about $15 \mathrm{~h}$. The product was recrystallized from $i$-propyl acetate, yielding crystals suitable for single-crystal X-ray diffraction, yield $11.7 \mathrm{~g}$ (92\%).

\section{Thermal analysis}

The thermal stability of the title compound was investigated in the solid state by thermogravimetric analysis (TGA) and by differential scanning calorimetry (DSC). Thermogravimetric analysis was performed on TA Instruments TGA in closed aluminium pans with one hole on the crucible under a nitrogen flow $\left(50 \mathrm{~mL} \mathrm{~min}^{-1}\right)$ with a heating rate of $10^{\circ} \mathrm{C} \mathrm{min}^{-1}$ in the temperature range $25-300^{\circ} \mathrm{C}$.

Thermogravimetric analysis does not reveal any weight loss during heating up to about $200^{\circ} \mathrm{C}$, whereupon a change in mass is observed that can be associated with the thermal decomposition of the sample (Fig. 6a). DSC analysis of (I) reveals two thermal events (Fig. $6 b$ ). The first endotherm at about $125^{\circ} \mathrm{C}$ suggests that the sample is experiencing a phase transition, as no weight loss can be observed on the corresponding TG curve in this temperature region. The second strong endotherm, observed on the DSC curve at about $210^{\circ} \mathrm{C}$, can be ascribed to the melting point of the new phase. Existence of a new, stable phase was confirmed via a PXRD experiment, where comparison of the powder patterns of the starting sample (I) and the one obtained by heating (I) at about $130^{\circ} \mathrm{C}$ for $17 \mathrm{~h}$ revealed significant differences (Fig. 7). Additional confirmation for this conclusion is found in the DSC curve of the material obtained after heating (I), where only one endothermic event can be observed, the one appearing at $210^{\circ} \mathrm{C}$ and corresponding to its melting point.

(a)
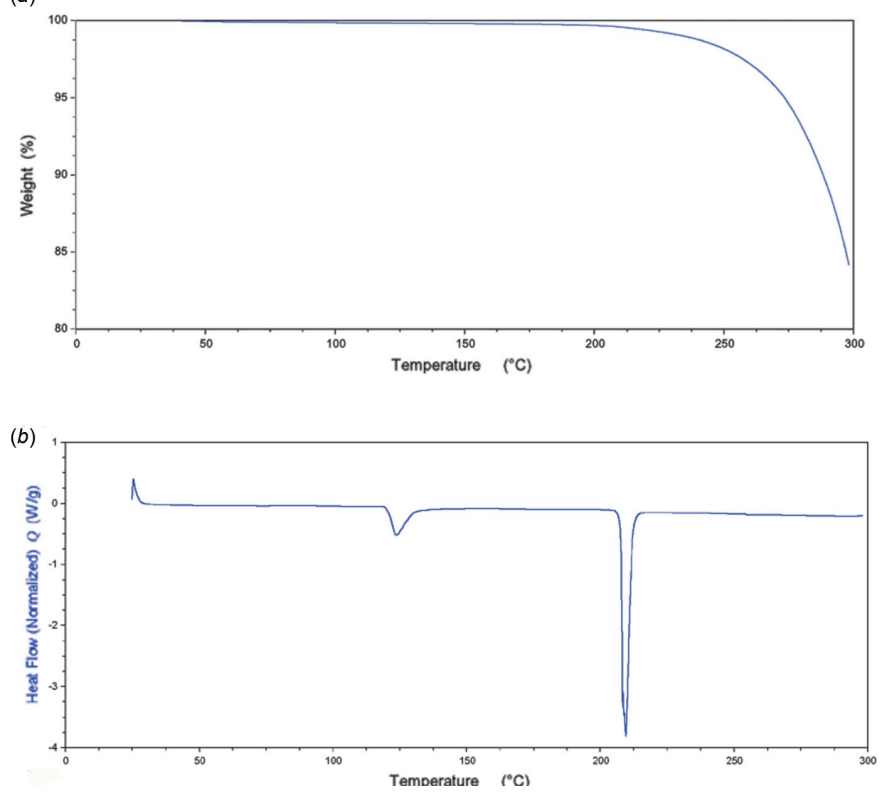

Figure 6

(a) TG curve of (I); (b) DSC curve of (I). 


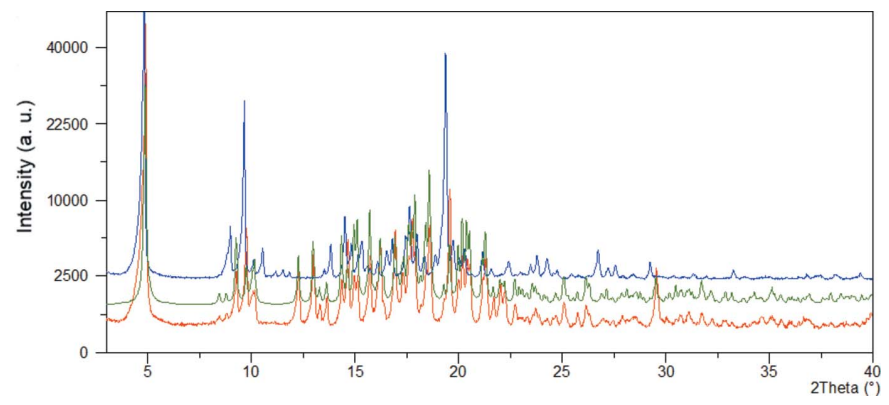

Figure 7

PXRD pattern of the bulk sample of I (red), simulated pattern for (I) (green), and PXRD pattern of the new phase obtained by heating (I) at about $130^{\circ} \mathrm{C}$ for $17 \mathrm{~h}$ (blue).

\section{IR spectroscopy}

The infrared (IR) spectrum of title compound was recorded by using the ATR (attenuated total reflectance) technique on a PerkinElmer Spectrum Two instrument. The spectrum of (I) displays a broad band positioned at $c a 2900 \mathrm{~cm}^{-1}$, which corresponds to $\mathrm{N}-\mathrm{H}$ stretching vibrations of the protonated amino group of the (3,5-dimethyladamantan-1-yl)ammonium cations superimposed with the $\mathrm{C}-\mathrm{H}$ stretching vibrations of the adamantane skeleton and methyl groups of the methanesulfonate anion (Fig. 8). The bands corresponding to the $\mathrm{S}-\mathrm{O}$ asymmetric and symmetric stretching modes appear at 1179 and $1042 \mathrm{~cm}^{-1}$, respectively (Başköse et al., 2012). The band at $780 \mathrm{~cm}^{-1}$ is associated with the $\mathrm{C}-\mathrm{S}$ stretching vibration, whereas the one at $540 \mathrm{~cm}^{-1}$ corresponds to the bending mode of the $\mathrm{SO}_{3}$ moiety (Başköse et al., 2012).

\section{Refinement}

Crystal data, data collection and structure refinement details are summarized in Table 2. Hydrogen atoms bonded to carbon atoms of the adamantane core were refined as riding with $\mathrm{C}-$ $\mathrm{H}=0.98 \AA$ for methine $\mathrm{C}$ atoms $(\mathrm{C} 7-\mathrm{H} 7, \mathrm{C} 19-\mathrm{H} 19$ and $\mathrm{C} 31-\mathrm{H} 31)$ and $\mathrm{C}-\mathrm{H}=0.97 \AA$ for the methylene $\mathrm{H}$ atoms, both with $U_{\text {iso }}(\mathrm{H})=1.2 U_{\text {eq }}(\mathrm{C})$. Hydrogen atoms bonded to carbon atoms of the methyl groups of both the memantine cations and the methanesulfonate anions were refined as rotating rigid groups with $\mathrm{C}-\mathrm{H}=0.96 \AA$ and $U_{\text {iso }}(\mathrm{H})=$ $1.5 U_{\text {eq }}(\mathrm{C})$. Hydrogen atoms bonded to nitrogen atoms were

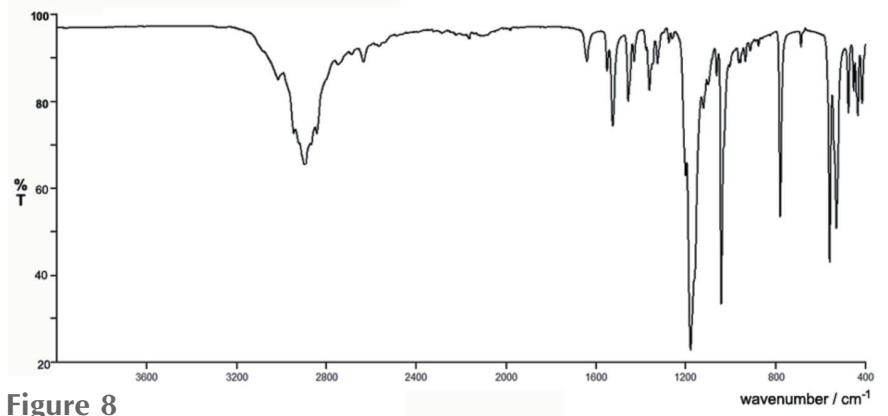

Figure 8

IR spectrum of the title compound.
Table 2

Experimental details.

\begin{tabular}{ll}
\hline Crystal data & \\
Chemical formula & $\mathrm{C}_{12} \mathrm{H}_{22} \mathrm{~N}^{+} \cdot \mathrm{CH}_{3} \mathrm{O}_{3} \mathrm{~S}^{-}$ \\
$M_{\mathrm{r}}$ & 275.40 \\
Crystal system, space group & Triclinic, $P \overline{1}$ \\
Temperature $(\mathrm{K})$ & 295 \\
$a, b, c(\AA)$ & $11.7761(2), 11.8731(2)$, \\
& $18.2788(3)$ \\
$\alpha, \beta, \gamma\left({ }^{\circ}\right)$ & $92.501(2), 94.696(2), 116.609(2)$ \\
$V\left(\AA^{3}\right)$ & $2268.09(8)$ \\
$Z$ & 6 \\
Radiation type & $\mathrm{Cu} \mathrm{K \alpha}$ \\
$\mu\left(\mathrm{mm}^{-1}\right)$ & 1.92 \\
Crystal size $(\mathrm{mm})$ & $0.32 \times 0.21 \times 0.11$ \\
& \\
Data collection & Oxford Diffraction Xcalibur \\
Diffractometer & Sapphire3 \\
& Multi-scan $(C r y s A l i s ~ P R O ;$ Rigaku, \\
Absorption correction & $2018)$ \\
& $0.200,1.000$ \\
$T_{\text {min }}, T_{\max }$ & $76762,8992,8048$ \\
No. of measured, independent and & \\
$\quad$ observed $[I>2 \sigma(I)]$ reflections & 0.062 \\
$R_{\text {int }}$ & 0.620 \\
(sin $\theta / \lambda)_{\text {max }}\left(\AA^{-1}\right)$ & \\
Refinement & \\
$R\left[F^{2}>2 \sigma\left(F^{2}\right)\right], w R\left(F^{2}\right), S$ & $0.047,0.133,1.06$ \\
No. of reflections & 8992 \\
No. of parameters & 523 \\
No. of restraints & 9 \\
$\mathrm{H}$-atom treatment & independent and constrained \\
& refinement \\
$\Delta \rho_{\text {max }}, \Delta \rho_{\text {min }}\left(\mathrm{e} \AA^{-3}\right)$ & $0.86,-0.53$ \\
\hline
\end{tabular}

Computer programs: CrysAlis PRO (Rigaku, 2018), SHELXT (Sheldrick, 2015a), SHELXL2018 (Sheldrick, 2015b) and Mercury (Macrae et al., 2008).

found in the difference-Fourier maps at final steps of the refinement and refined with $U_{\text {iso }}(\mathrm{H})=1.2 U_{\text {eq }}(\mathrm{N})$. Their coordinates were refined independently, but $\mathrm{N}-\mathrm{H}$ distances were restrained to 0.89 (2) $\AA$.

\section{Acknowledgements}

MT and EM acknowledge PLIVA for financial support.

\section{References}

Başköse, U. C., Bayarı, S. H., Sağlam, S. \& Özışı, H. (2012). Open Chem. 10, 395-406.

Groom, C. R., Bruno, I. J., Lightfoot, M. P. \& Ward, S. C. (2016). Acta Cryst. B72, 171-179.

Hostaš, J., Sigwalt, D., Šekutor, M., Ajani, H., Dubecký, M., Řezáč, J., Zavalij, P. Y., Cao, L., Wohlschlager, C., Mlinarić-Majerski, K., Isaacs, L., Glaser, R. \& Hobza, P. (2016). Chem. Eur. J. 22, 1722617238.

Lou, W.-J., Hu, X.-R. \& Gu, J.-M. (2009). Acta Cryst. E65, o2191.

Macrae, C. F., Bruno, I. J., Chisholm, J. A., Edgington, P. R., McCabe, P., Pidcock, E., Rodriguez-Monge, L., Taylor, R., van de Streek, J. \& Wood, P. A. (2008). J. Appl. Cryst. 41, 466-470.

McInnes, F. J., Anthony, N. G., Kennedy, A. R. \& Wheate, N. J. (2010). Org. Biomol. Chem. 8, 765-773.

Parsons, C. G., Danysz, W., Dekundy, A. \& Pulte, I. (2013). Neurotox. Res. 24, 358-369.

Rammes, G., Danysz, W. \& Parsons, C. G. (2008). Curr. Neuropharmacol. 6, 55-78. 
Reisberg, B., Doody, R., Stöffler, A., Schmitt, F., Ferris, S. \& Möbius, H. J. (2003). N. Engl. J. Med. 348, 1333-1341.

Rigaku (2018). CrysAlis PRO. Rigaku Oxford Diffraction Ltd, Yarnton, England.

Sheldrick, G. M. (2015a). Acta Cryst. A71, 3-8.

Sheldrick, G. M. (2015b). Acta Cryst. C71, 3-8.
Spackman, M. A. \& Jayatilaka, D. (2009). CrystEngComm, 11, 19-32. Tkachev, V. V., Tkacheva, N. S. \& Kazachenko, V. P. (2017). Zh. Strukt. Khim. (Russ. J. Struct. Chem.), 58, 615-617.

Turner, M. J., McKinnon, J. J., Wolff, S. K., Grimwood, D. J., Spackman, P. R., Jayatilaka, D. \& Spackman, M. A. (2017). CrystalExplorer17. University of Western Australia. 


\section{supporting information}

Acta Cryst. (2019). E75, 1274-1279 [https://doi.org/10.1107/S2056989019009988]

\section{(3,5-Dimethyladamantan-1-yl)ammonium methanesulfonate (memantinium mesylate): synthesis, structure and solid-state properties}

\section{Mihaela Tuksar, Mirta Rubčić and Ernest Meštrović}

\section{Computing details}

Data collection: CrysAlis PRO (Rigaku, 2018); cell refinement: CrysAlis PRO (Rigaku, 2018); data reduction: CrysAlis PRO (Rigaku, 2018); program(s) used to solve structure: SHELXT (Sheldrick, 2015a); program(s) used to refine structure: SHELXL2018 (Sheldrick, 2015b); molecular graphics: Mercury (Macrae et al., 2008); software used to prepare material for publication: SHELXL2018 (Sheldrick, 2015b).

(3,5-Dimethyladamantan-1-yl)ammonium methanesulfonate

\section{Crystal data}

$\mathrm{C}_{12} \mathrm{H}_{22} \mathrm{~N}^{+} \cdot \mathrm{CH}_{3} \mathrm{O}_{3} \mathrm{~S}^{-}$

$M_{r}=275.40$

Triclinic, $P \overline{1}$

$a=11.7761$ (2) $\AA$

$b=11.8731(2) \AA$

$c=18.2788(3) \AA$

$\alpha=92.501(2)^{\circ}$

$\beta=94.696(2)^{\circ}$

$\gamma=116.609(2)^{\circ}$

$V=2268.09(8) \AA^{3}$

\section{Data collection}

Oxford Diffraction Xcalibur Sapphire3 diffractometer

Radiation source: fine-focus sealed X-ray tube, Enhance $(\mathrm{Cu}) \mathrm{X}$-ray Source

Graphite monochromator

Detector resolution: 16.1285 pixels $\mathrm{mm}^{-1}$

$\omega$ scans

Absorption correction: multi-scan

(CrysAlis PRO; Rigaku, 2018)

\section{Refinement}

Refinement on $F^{2}$

Least-squares matrix: full

$R\left[F^{2}>2 \sigma\left(F^{2}\right)\right]=0.047$

$w R\left(F^{2}\right)=0.133$

$S=1.06$

8992 reflections

523 parameters
$Z=6$

$F(000)=900$

$D_{\mathrm{x}}=1.210 \mathrm{Mg} \mathrm{m}^{-3}$

$\mathrm{Cu} K \alpha$ radiation, $\lambda=1.54184 \AA$

Cell parameters from 30823 reflections

$\theta=5.1-72.7^{\circ}$

$\mu=1.92 \mathrm{~mm}^{-1}$

$T=295 \mathrm{~K}$

Prism, colorless

$0.32 \times 0.21 \times 0.11 \mathrm{~mm}$

$T_{\min }=0.200, T_{\max }=1.000$

76762 measured reflections

8992 independent reflections

8048 reflections with $I>2 \sigma(I)$

$R_{\text {int }}=0.062$

$\theta_{\max }=72.9^{\circ}, \theta_{\min }=4.2^{\circ}$

$h=-13 \rightarrow 14$

$k=-14 \rightarrow 14$

$l=-22 \rightarrow 22$

9 restraints

Primary atom site location: dual

Hydrogen site location: mixed

$\mathrm{H}$ atoms treated by a mixture of independent and constrained refinement

$w=1 /\left[\sigma^{2}\left(F_{0}^{2}\right)+(0.0822 P)^{2}+0.429 P\right]$ where $P=\left(F_{\mathrm{o}}{ }^{2}+2 F_{\mathrm{c}}{ }^{2}\right) / 3$ 
$(\Delta / \sigma)_{\max }<0.001$

$\Delta \rho_{\max }=0.86$ e $\AA^{-3}$

$$
\Delta \rho_{\min }=-0.53 \text { e } \AA^{-3}
$$

Special details

Geometry. All esds (except the esd in the dihedral angle between two 1.s. planes) are estimated using the full covariance matrix. The cell esds are taken into account individually in the estimation of esds in distances, angles and torsion angles; correlations between esds in cell parameters are only used when they are defined by crystal symmetry. An approximate (isotropic) treatment of cell esds is used for estimating esds involving l.s. planes.

Fractional atomic coordinates and isotropic or equivalent isotropic displacement parameters $\left(\AA^{2}\right)$

\begin{tabular}{|c|c|c|c|c|}
\hline & $x$ & $y$ & $z$ & $U_{\text {iso }} * / U_{\text {eq }}$ \\
\hline S1 & $0.63651(4)$ & $0.78463(4)$ & $0.39086(2)$ & $0.04527(12)$ \\
\hline S2 & $0.67423(4)$ & $0.34333(4)$ & $0.38657(2)$ & $0.04606(12)$ \\
\hline S3 & $0.12114(4)$ & $0.83219(4)$ & $0.39052(2)$ & $0.04759(12)$ \\
\hline $\mathrm{O} 2$ & $0.51832(13)$ & $0.79458(13)$ & $0.39206(8)$ & $0.0608(3)$ \\
\hline O6 & $0.66922(13)$ & $0.21916(12)$ & $0.38512(8)$ & $0.0615(3)$ \\
\hline $\mathrm{O} 3$ & $0.63630(13)$ & $0.68285(13)$ & $0.43196(8)$ & 0.0637 (4) \\
\hline O9 & $0.22683(12)$ & $0.95327(12)$ & $0.38131(8)$ & 0.0597 (3) \\
\hline N2 & $0.46247(14)$ & $-0.01597(13)$ & $0.33925(7)$ & $0.0433(3)$ \\
\hline $\mathrm{H} 2 \mathrm{~A}$ & $0.4793(18)$ & $-0.0755(16)$ & $0.3563(11)$ & $0.052 *$ \\
\hline $\mathrm{H} 2 \mathrm{~B}$ & $0.3914(16)$ & $-0.0237(19)$ & $0.3549(11)$ & $0.052 *$ \\
\hline $\mathrm{H} 2 \mathrm{C}$ & $0.5300(16)$ & $0.0593(15)$ & $0.3524(11)$ & $0.052 *$ \\
\hline N3 & $0.84089(14)$ & $0.66545(13)$ & $0.50755(7)$ & $0.0443(3)$ \\
\hline $\mathrm{H} 3 \mathrm{~A}$ & $0.7713(16)$ & $0.6768(19)$ & $0.4943(11)$ & $0.053^{*}$ \\
\hline $\mathrm{H} 3 \mathrm{~B}$ & $0.8237(19)$ & $0.5892(15)$ & $0.4864(11)$ & $0.053^{*}$ \\
\hline $\mathrm{H} 3 \mathrm{C}$ & $0.9054(17)$ & $0.7263(17)$ & $0.4886(11)$ & $0.053^{*}$ \\
\hline O5 & $0.78864(13)$ & $0.43837(13)$ & $0.42985(9)$ & $0.0681(4)$ \\
\hline O8 & $0.03123(14)$ & $0.84536(14)$ & $0.43564(9)$ & $0.0701(4)$ \\
\hline $\mathrm{O} 1$ & $0.66929(16)$ & $0.77755(15)$ & $0.31635(8)$ & 0.0689 (4) \\
\hline N1 & $0.79465(15)$ & $0.63192(15)$ & $0.27752(8)$ & $0.0492(3)$ \\
\hline $\mathrm{H} 1 \mathrm{~A}$ & $0.8792(15)$ & $0.6704(19)$ & $0.2924(11)$ & $0.059 *$ \\
\hline $\mathrm{H} 1 \mathrm{~B}$ & $0.7552(19)$ & $0.6784(19)$ & $0.2896(11)$ & $0.059^{*}$ \\
\hline $\mathrm{H} 1 \mathrm{C}$ & $0.7541(19)$ & $0.5562(16)$ & $0.2945(11)$ & $0.059^{*}$ \\
\hline $\mathrm{O} 4$ & $0.65613(18)$ & $0.37874(16)$ & $0.31366(8)$ & $0.0799(4)$ \\
\hline $\mathrm{O} 7$ & $0.06024(15)$ & $0.75862(18)$ & $0.32090(8)$ & $0.0807(5)$ \\
\hline $\mathrm{C} 1$ & $0.78006(15)$ & $0.61136(14)$ & $0.19499(8)$ & $0.0403(3)$ \\
\hline $\mathrm{C} 13$ & $0.44439(15)$ & $-0.03377(14)$ & $0.25673(8)$ & $0.0410(3)$ \\
\hline $\mathrm{C} 21$ & $0.57125(16)$ & $-0.00684(16)$ & $0.22910(9)$ & $0.0466(3)$ \\
\hline $\mathrm{H} 21 \mathrm{~A}$ & 0.602030 & -0.063954 & 0.248806 & $0.056^{*}$ \\
\hline $\mathrm{H} 21 \mathrm{~B}$ & 0.633874 & 0.079104 & 0.245897 & $0.056^{*}$ \\
\hline $\mathrm{C} 2$ & $0.85215(15)$ & $0.73767(14)$ & $0.16319(9)$ & 0.0437 (3) \\
\hline $\mathrm{H} 2 \mathrm{D}$ & 0.941858 & 0.775209 & 0.182302 & $0.052^{*}$ \\
\hline $\mathrm{H} 2 \mathrm{E}$ & 0.818326 & 0.795085 & 0.177964 & $0.052 *$ \\
\hline $\mathrm{C} 25$ & $0.86750(14)$ & $0.67267(14)$ & $0.58989(8)$ & 0.0388 (3) \\
\hline $\mathrm{C} 8$ & $0.63814(16)$ & $0.55422(17)$ & $0.16706(9)$ & 0.0495 (4) \\
\hline H8A & 0.603732 & 0.611128 & 0.181834 & $0.059^{*}$ \\
\hline H8B & 0.591700 & 0.474521 & 0.187850 & $0.059^{*}$ \\
\hline $\mathrm{C} 14$ & $0.39642(17)$ & $0.05787(16)$ & $0.22769(9)$ & $0.0465(4)$ \\
\hline
\end{tabular}




\begin{tabular}{|c|c|c|c|c|}
\hline H14A & 0.458103 & 0.144183 & 0.244420 & $0.056^{*}$ \\
\hline H14B & 0.316197 & 0.041308 & 0.246425 & $0.056^{*}$ \\
\hline C33 & $0.74579(15)$ & $0.58585(15)$ & $0.62134(9)$ & $0.0465(4)$ \\
\hline $\mathrm{H} 33 \mathrm{~A}$ & 0.716038 & 0.499680 & 0.600214 & $0.056^{*}$ \\
\hline H33B & 0.679657 & 0.611394 & 0.608577 & $0.056^{*}$ \\
\hline $\mathrm{C} 3$ & $0.83830(16)$ & $0.71802(15)$ & $0.07895(9)$ & $0.0461(3)$ \\
\hline C9 & $0.83407(18)$ & $0.52124(16)$ & $0.17324(9)$ & 0.0494 (4) \\
\hline H9A & 0.789149 & 0.441714 & 0.194516 & $0.059 *$ \\
\hline H9B & 0.923813 & 0.557457 & 0.192103 & $0.059^{*}$ \\
\hline $\mathrm{C} 29$ & $0.77116(18)$ & $0.59194(16)$ & $0.70548(10)$ & $0.0515(4)$ \\
\hline $\mathrm{C} 17$ & $0.55484(18)$ & $-0.02435(18)$ & $0.14428(9)$ & $0.0518(4)$ \\
\hline $\mathrm{C} 20$ & $0.34628(19)$ & $-0.17022(16)$ & $0.23234(10)$ & $0.0550(4)$ \\
\hline $\mathrm{H} 20 \mathrm{~A}$ & 0.265548 & -0.187900 & 0.250751 & $0.066^{*}$ \\
\hline H20B & 0.376024 & -0.228155 & 0.251938 & $0.066^{*}$ \\
\hline C7 & $0.62339(17)$ & $0.53292(18)$ & $0.08302(10)$ & $0.0570(4)$ \\
\hline H7 & 0.532571 & 0.496120 & 0.064282 & $0.068^{*}$ \\
\hline $\mathrm{C} 10$ & $0.69578(17)$ & $0.65902(18)$ & $0.05044(10)$ & $0.0551(4)$ \\
\hline $\mathrm{H} 10 \mathrm{~A}$ & 0.661208 & 0.716286 & 0.064169 & $0.066^{*}$ \\
\hline H10B & 0.685028 & 0.645408 & -0.002913 & $0.066^{*}$ \\
\hline $\mathrm{C} 27$ & $0.94396(19)$ & $0.81789(16)$ & $0.70574(10)$ & $0.0550(4)$ \\
\hline $\mathrm{C} 28$ & 0.82115 (19) & $0.72963(17)$ & $0.73701(10)$ & $0.0547(4)$ \\
\hline $\mathrm{H} 28 \mathrm{~A}$ & 0.756156 & 0.757187 & 0.725515 & $0.066^{*}$ \\
\hline $\mathrm{H} 28 \mathrm{~B}$ & 0.837695 & 0.734841 & 0.790224 & $0.066^{*}$ \\
\hline C32 & $0.97039(18)$ & $0.63053(19)$ & $0.60735(10)$ & $0.0533(4)$ \\
\hline $\mathrm{H} 32 \mathrm{~A}$ & 1.047882 & 0.685745 & 0.587058 & $0.064 *$ \\
\hline H32B & 0.941860 & 0.544971 & 0.585529 & $0.064 *$ \\
\hline $\mathrm{C} 26$ & $0.91308(17)$ & $0.80841(14)$ & $0.62144(9)$ & $0.0480(4)$ \\
\hline $\mathrm{H} 26 \mathrm{~A}$ & 0.847120 & 0.834588 & 0.609658 & $0.058 *$ \\
\hline H26B & 0.988817 & 0.864055 & 0.599813 & $0.058^{*}$ \\
\hline $\mathrm{C} 15$ & $0.37718(19)$ & $0.04088(19)$ & $0.14298(10)$ & $0.0541(4)$ \\
\hline $\mathrm{C} 4$ & $0.89072(19)$ & $0.62571(18)$ & $0.05718(10)$ & $0.0547(4)$ \\
\hline $\mathrm{H} 4 \mathrm{~A}$ & 0.980834 & 0.662137 & 0.075195 & $0.066^{*}$ \\
\hline $\mathrm{H} 4 \mathrm{~B}$ & 0.882463 & 0.612470 & 0.003875 & $0.066^{*}$ \\
\hline $\mathrm{C} 16$ & $0.50501(19)$ & $0.06612(19)$ & $0.11542(10)$ & $0.0560(4)$ \\
\hline H16A & 0.494476 & 0.056705 & 0.061962 & $0.067 *$ \\
\hline H16B & 0.567400 & 0.152538 & 0.131348 & $0.067 *$ \\
\hline $\mathrm{C} 5$ & $0.8188(2)$ & $0.49778(17)$ & $0.08899(10)$ & $0.0581(4)$ \\
\hline $\mathrm{C} 31$ & $0.99688(19)$ & $0.6358(2)$ & $0.69101(11)$ & $0.0613(5)$ \\
\hline H31 & 1.062627 & 0.608260 & 0.703034 & $0.074 *$ \\
\hline C34 & $1.04425(19)$ & $0.7718(2)$ & $0.72352(11)$ & $0.0667(5)$ \\
\hline H34A & 1.122481 & 0.826226 & 0.703591 & $0.080 *$ \\
\hline H34B & 1.063191 & 0.776712 & 0.776554 & $0.080^{*}$ \\
\hline $\mathrm{C} 18$ & $0.4547(2)$ & $-0.16083(19)$ & $0.11936(11)$ & $0.0642(5)$ \\
\hline $\mathrm{H} 18 \mathrm{~A}$ & 0.442643 & -0.173304 & 0.065954 & $0.077 *$ \\
\hline H18B & 0.484759 & -0.219250 & 0.137810 & $0.077^{*}$ \\
\hline C30 & $0.8754(2)$ & $0.55030(19)$ & $0.72313(11)$ & $0.0622(5)$ \\
\hline $\mathrm{H} 30 \mathrm{~A}$ & 0.893369 & 0.553496 & 0.776130 & $0.075^{*}$ \\
\hline $\mathrm{H} 30 \mathrm{~B}$ & 0.845513 & 0.463685 & 0.702762 & $0.075^{*}$ \\
\hline
\end{tabular}




\begin{tabular}{|c|c|c|c|c|}
\hline $\mathrm{C} 22$ & $0.2800(2)$ & $-0.0963(2)$ & $0.11844(11)$ & $0.0669(5)$ \\
\hline $\mathrm{H} 22 \mathrm{~A}$ & 0.265956 & -0.108476 & 0.065038 & $0.080 *$ \\
\hline $\mathrm{H} 22 \mathrm{~B}$ & 0.199032 & -0.114068 & 0.136608 & $0.080 *$ \\
\hline $\mathrm{C} 11$ & $0.9113(2)$ & 0.84445 (19) & $0.04648(12)$ & $0.0637(5)$ \\
\hline H11A & 0.906209 & 0.830026 & -0.006045 & $0.096 *$ \\
\hline H11B & 0.999280 & 0.882959 & 0.067192 & $0.096^{*}$ \\
\hline $\mathrm{H} 11 \mathrm{C}$ & 0.874229 & 0.899532 & 0.057953 & $0.096^{*}$ \\
\hline C6 & $0.6764(2)$ & $0.44274(18)$ & $0.06038(11)$ & $0.0675(6)$ \\
\hline H6A & 0.665622 & 0.428149 & 0.007073 & $0.081 *$ \\
\hline H6B & 0.629446 & 0.362181 & 0.080179 & $0.081 *$ \\
\hline C19 & $0.3283(2)$ & $-0.18780(18)$ & $0.14795(11)$ & $0.0624(5)$ \\
\hline H19 & 0.265373 & -0.274972 & 0.131457 & $0.075 *$ \\
\hline C37 & $0.7589(2)$ & $0.9253(2)$ & $0.43596(13)$ & $0.0725(6)$ \\
\hline $\mathrm{H} 37 \mathrm{~A}$ & 0.839530 & 0.923855 & 0.434650 & $0.109 *$ \\
\hline H37B & 0.743534 & 0.933165 & 0.486261 & $0.109^{*}$ \\
\hline $\mathrm{H} 37 \mathrm{C}$ & 0.760564 & 0.996040 & 0.411638 & $0.109^{*}$ \\
\hline $\mathrm{C} 24$ & $0.6820(2)$ & $0.0025(3)$ & $0.11616(13)$ & $0.0750(6)$ \\
\hline $\mathrm{H} 24 \mathrm{~A}$ & 0.716007 & -0.048908 & 0.139055 & $0.112 *$ \\
\hline H24B & 0.741106 & 0.090204 & 0.128023 & $0.112 *$ \\
\hline $\mathrm{H} 24 \mathrm{C}$ & 0.669158 & -0.016810 & 0.063679 & $0.112^{*}$ \\
\hline C39 & $0.1879(3)$ & $0.7482(2)$ & $0.43919(13)$ & $0.0772(6)$ \\
\hline $\mathrm{H} 39 \mathrm{~A}$ & 0.121442 & 0.666667 & 0.446964 & $0.116^{*}$ \\
\hline H39B & 0.230009 & 0.794210 & 0.485935 & $0.116^{*}$ \\
\hline $\mathrm{H} 39 \mathrm{C}$ & 0.248720 & 0.737812 & 0.411344 & $0.116^{*}$ \\
\hline $\mathrm{C} 23$ & $0.3304(3)$ & $0.1337(3)$ & $0.11357(13)$ & $0.0826(7)$ \\
\hline $\mathrm{H} 23 \mathrm{~A}$ & 0.390720 & 0.218550 & 0.131858 & $0.124 *$ \\
\hline H23B & 0.248716 & 0.114611 & 0.129763 & $0.124^{*}$ \\
\hline $\mathrm{H} 23 \mathrm{C}$ & 0.322531 & 0.126083 & 0.060676 & $0.124^{*}$ \\
\hline C36 & $0.6483(2)$ & $0.5065(2)$ & $0.73711(14)$ & $0.0810(7)$ \\
\hline $\mathrm{H} 36 \mathrm{~A}$ & 0.614309 & 0.422261 & 0.713577 & $0.122 *$ \\
\hline H36B & 0.586811 & 0.538270 & 0.728493 & $0.122 *$ \\
\hline $\mathrm{H} 36 \mathrm{C}$ & 0.666388 & 0.505295 & 0.789161 & $0.122 *$ \\
\hline C38 & $0.5456(2)$ & $0.3353(3)$ & $0.43141(15)$ & $0.0786(6)$ \\
\hline $\mathrm{H} 38 \mathrm{~A}$ & 0.541871 & 0.414355 & 0.430089 & $0.118^{*}$ \\
\hline H38B & 0.556891 & 0.318705 & 0.481714 & $0.118^{*}$ \\
\hline $\mathrm{H} 38 \mathrm{C}$ & 0.467537 & 0.268600 & 0.407036 & $0.118^{*}$ \\
\hline C35 & $0.9899(3)$ & $0.9543(2)$ & $0.73805(14)$ & $0.0888(8)$ \\
\hline $\mathrm{H} 35 \mathrm{~A}$ & 0.924801 & 0.980266 & 0.725943 & $0.133^{*}$ \\
\hline H35B & 1.066459 & 1.008865 & 0.717829 & $0.133^{*}$ \\
\hline $\mathrm{H} 35 \mathrm{C}$ & 1.007226 & 0.959284 & 0.790653 & $0.133^{*}$ \\
\hline $\mathrm{C} 12$ & $0.8726(4)$ & $0.4071(3)$ & $0.06721(16)$ & $0.0966(9)$ \\
\hline $\mathrm{H} 12 \mathrm{~A}$ & 0.860777 & 0.390739 & 0.014533 & $0.145^{*}$ \\
\hline H12B & 0.828734 & 0.329128 & 0.089069 & $0.145^{*}$ \\
\hline $\mathrm{H} 12 \mathrm{C}$ & 0.962179 & 0.444489 & 0.084328 & $0.145^{*}$ \\
\hline
\end{tabular}


Atomic displacement parameters $\left(\AA^{2}\right)$

\begin{tabular}{|c|c|c|c|c|c|c|}
\hline & $U^{11}$ & $U^{22}$ & $U^{33}$ & $U^{12}$ & $U^{13}$ & $U^{23}$ \\
\hline $\mathrm{S} 1$ & $0.0487(2)$ & $0.0458(2)$ & $0.0460(2)$ & $0.02573(18)$ & $0.00387(16)$ & $0.00512(15)$ \\
\hline $\mathrm{S} 2$ & $0.0469(2)$ & $0.0452(2)$ & $0.0465(2)$ & $0.02134(17)$ & $0.00555(16)$ & $0.00208(15)$ \\
\hline $\mathrm{S} 3$ & $0.0452(2)$ & $0.0508(2)$ & $0.0449(2)$ & $0.01982(18)$ & $0.00683(16)$ & $0.00448(16)$ \\
\hline $\mathrm{O} 2$ & $0.0593(8)$ & $0.0671(8)$ & $0.0715(8)$ & $0.0403(7)$ & $0.0124(6)$ & $0.0197(6)$ \\
\hline O6 & $0.0610(8)$ & $0.0462(6)$ & $0.0745(9)$ & $0.0241(6)$ & $-0.0017(6)$ & $-0.0025(6)$ \\
\hline $\mathrm{O} 3$ & $0.0582(8)$ & $0.0571(7)$ & $0.0800(9)$ & $0.0301(6)$ & $-0.0012(7)$ & $0.0199(6)$ \\
\hline O9 & $0.0518(7)$ & $0.0555(7)$ & $0.0734(8)$ & $0.0235(6)$ & $0.0180(6)$ & $0.0126(6)$ \\
\hline N2 & $0.0448(7)$ & $0.0447(7)$ & $0.0426(7)$ & $0.0218(6)$ & $0.0052(6)$ & $0.0063(5)$ \\
\hline N3 & $0.0466(8)$ & $0.0460(7)$ & $0.0408(7)$ & $0.0222(6)$ & $0.0014(6)$ & $0.0012(5)$ \\
\hline O5 & $0.0549(8)$ & $0.0566(7)$ & $0.0888(10)$ & $0.0262(6)$ & $-0.0052(7)$ & $-0.0202(7)$ \\
\hline O8 & $0.0662(9)$ & $0.0684(8)$ & $0.0819(10)$ & $0.0310(7)$ & $0.0342(7)$ & $0.0145(7)$ \\
\hline $\mathrm{O} 1$ & $0.0861(10)$ & $0.0835(9)$ & $0.0517(7)$ & $0.0504(8)$ & $0.0150(7)$ & $0.0031(6)$ \\
\hline N1 & $0.0524(8)$ & $0.0561(8)$ & $0.0377(7)$ & $0.0238(7)$ & $0.0040(6)$ & $0.0008(6)$ \\
\hline $\mathrm{O} 4$ & $0.1053(12)$ & $0.0754(10)$ & $0.0541(8)$ & $0.0353(9)$ & $0.0109(8)$ & $0.0192(7)$ \\
\hline O7 & $0.0631(9)$ & $0.1011(12)$ & $0.0585(8)$ & $0.0245(8)$ & $-0.0072(7)$ & $-0.0159(8)$ \\
\hline $\mathrm{C} 1$ & $0.0414(8)$ & $0.0436(7)$ & $0.0347(7)$ & $0.0188(6)$ & $0.0020(6)$ & $0.0012(5)$ \\
\hline C13 & $0.0452(8)$ & $0.0406(7)$ & $0.0389(7)$ & $0.0213(6)$ & $0.0030(6)$ & $0.0042(6)$ \\
\hline $\mathrm{C} 21$ & $0.0471(9)$ & $0.0523(9)$ & $0.0459(8)$ & $0.0277(7)$ & $0.0041(7)$ & $0.0049(6)$ \\
\hline $\mathrm{C} 2$ & $0.0418(8)$ & $0.0392(7)$ & $0.0460(8)$ & $0.0157(6)$ & $0.0002(6)$ & $0.0008(6)$ \\
\hline $\mathrm{C} 25$ & $0.0394(8)$ & $0.0398(7)$ & $0.0388(7)$ & $0.0199(6)$ & $0.0019(6)$ & $0.0019(5)$ \\
\hline $\mathrm{C} 8$ & $0.0396(8)$ & $0.0561(9)$ & $0.0469(9)$ & $0.0163(7)$ & $0.0059(7)$ & $0.0045(7)$ \\
\hline $\mathrm{C} 14$ & $0.0502(9)$ & $0.0506(8)$ & $0.0477(8)$ & $0.0306(7)$ & $0.0058(7)$ & $0.0076(7)$ \\
\hline C33 & $0.0417(8)$ & $0.0416(8)$ & $0.0499(9)$ & $0.0141(7)$ & $0.0029(7)$ & $0.0008(6)$ \\
\hline $\mathrm{C} 3$ & $0.0453(8)$ & $0.0458(8)$ & $0.0436(8)$ & $0.0175(7)$ & $0.0030(6)$ & $0.0079(6)$ \\
\hline C9 & $0.0583(10)$ & $0.0490(8)$ & $0.0482(9)$ & $0.0293(8)$ & $0.0122(7)$ & $0.0101(7)$ \\
\hline C29 & $0.0529(10)$ & $0.0469(8)$ & $0.0500(9)$ & $0.0174(7)$ & $0.0119(7)$ & $0.0068(7)$ \\
\hline C17 & $0.0551(10)$ & $0.0605(10)$ & $0.0454(8)$ & $0.0310(8)$ & $0.0075(7)$ & $0.0030(7)$ \\
\hline $\mathrm{C} 20$ & $0.0577(10)$ & $0.0423(8)$ & $0.0570(10)$ & $0.0159(8)$ & $0.0051(8)$ & $0.0049(7)$ \\
\hline C7 & $0.0425(9)$ & $0.0619(10)$ & $0.0463(9)$ & $0.0079(8)$ & $-0.0052(7)$ & $0.0014(7)$ \\
\hline C10 & $0.0501(10)$ & $0.0640(10)$ & $0.0459(9)$ & $0.0222(8)$ & $-0.0034(7)$ & $0.0089(7)$ \\
\hline $\mathrm{C} 27$ & $0.0631(11)$ & $0.0425(8)$ & $0.0474(9)$ & $0.0149(8)$ & $0.0012(8)$ & $-0.0042(7)$ \\
\hline $\mathrm{C} 28$ & $0.0646(11)$ & $0.0566(10)$ & $0.0460(9)$ & $0.0300(9)$ & $0.0108(8)$ & $-0.0003(7)$ \\
\hline $\mathrm{C} 32$ & $0.0534(10)$ & $0.0683(11)$ & $0.0518(9)$ & $0.0396(9)$ & $0.0055(7)$ & $0.0049(8)$ \\
\hline $\mathrm{C} 26$ & $0.0532(9)$ & $0.0369(7)$ & $0.0486(9)$ & $0.0162(7)$ & $0.0029(7)$ & $0.0024(6)$ \\
\hline $\mathrm{C} 15$ & $0.0604(10)$ & $0.0673(11)$ & $0.0453(9)$ & $0.0385(9)$ & $0.0020(7)$ & $0.0099(7)$ \\
\hline $\mathrm{C} 4$ & $0.0619(11)$ & $0.0598(10)$ & $0.0468(9)$ & $0.0292(9)$ & $0.0168(8)$ & $0.0087(7)$ \\
\hline $\mathrm{C} 16$ & $0.0650(11)$ & $0.0644(10)$ & $0.0447(8)$ & $0.0335(9)$ & $0.0100(8)$ & $0.0131(7)$ \\
\hline $\mathrm{C} 5$ & 0.0807 (13) & $0.0510(9)$ & $0.0505(9)$ & $0.0346(9)$ & $0.0211(9)$ & $0.0042(7)$ \\
\hline C31 & $0.0606(11)$ & $0.0860(13)$ & $0.0547(10)$ & 0.0495 (11) & $-0.0007(8)$ & $0.0110(9)$ \\
\hline C34 & $0.0477(10)$ & $0.0842(14)$ & $0.0510(10)$ & $0.0175(10)$ & $-0.0082(8)$ & $0.0000(9)$ \\
\hline C18 & $0.0839(14)$ & $0.0612(11)$ & $0.0528(10)$ & 0.0394 (11) & $0.0032(9)$ & $-0.0076(8)$ \\
\hline $\mathrm{C} 30$ & $0.0843(14)$ & $0.0586(10)$ & $0.0519(10)$ & $0.0391(10)$ & $0.0062(9)$ & $0.0132(8)$ \\
\hline $\mathrm{C} 22$ & $0.0564(11)$ & $0.0861(14)$ & $0.0511(10)$ & $0.0291(10)$ & $-0.0084(8)$ & $-0.0019(9)$ \\
\hline $\mathrm{C} 11$ & $0.0627(11)$ & $0.0570(10)$ & $0.0664(11)$ & $0.0210(9)$ & 0.0078 (9) & $0.0226(9)$ \\
\hline C6 & $0.0829(14)$ & 0.0473 (9) & $0.0471(9)$ & $0.0088(9)$ & $0.0043(9)$ & $-0.0066(7)$ \\
\hline
\end{tabular}




\begin{tabular}{lllllll} 
C19 & $0.0633(11)$ & $0.0510(10)$ & $0.0567(10)$ & $0.0146(9)$ & $-0.0034(9)$ & $-0.0094(8)$ \\
C37 & $0.0709(13)$ & $0.0549(11)$ & $0.0742(13)$ & $0.0149(10)$ & $0.0018(11)$ & $-0.0047(9)$ \\
C24 & $0.0710(14)$ & $0.1042(17)$ & $0.0612(12)$ & $0.0486(13)$ & $0.0164(10)$ & $0.0057(11)$ \\
C39 & $0.1087(19)$ & $0.0742(13)$ & $0.0702(13)$ & $0.0590(14)$ & $0.0121(12)$ & $0.0177(11)$ \\
C23 & $0.1070(19)$ & $0.1152(19)$ & $0.0631(13)$ & $0.0822(17)$ & $0.0089(12)$ & $0.0256(12)$ \\
C36 & $0.0736(15)$ & $0.0771(14)$ & $0.0745(14)$ & $0.0141(12)$ & $0.0283(12)$ & $0.0176(11)$ \\
C38 & $0.0623(13)$ & $0.0978(17)$ & $0.0868(16)$ & $0.0438(13)$ & $0.0230(11)$ & $0.0076(13)$ \\
C35 & $0.122(2)$ & $0.0487(11)$ & $0.0681(13)$ & $0.0174(12)$ & $0.0026(13)$ & $-0.0133(9)$ \\
C12 & $0.157(3)$ & $0.0811(16)$ & $0.0877(17)$ & $0.0780(18)$ & $0.0545(18)$ & $0.0141(13)$ \\
\hline
\end{tabular}

Geometric parameters $\left(\AA,{ }^{\circ}\right)$

\begin{tabular}{|c|c|c|c|}
\hline $\mathrm{S} 1-\mathrm{O} 3$ & $1.4497(13)$ & $\mathrm{C} 10-\mathrm{H} 10 \mathrm{~A}$ & 0.9700 \\
\hline $\mathrm{S} 1-\mathrm{O} 2$ & $1.4503(13)$ & $\mathrm{C} 10-\mathrm{H} 10 \mathrm{~B}$ & 0.9700 \\
\hline $\mathrm{S} 1-\mathrm{O} 1$ & $1.4548(14)$ & $\mathrm{C} 27-\mathrm{C} 34$ & $1.527(3)$ \\
\hline $\mathrm{S} 1-\mathrm{C} 37$ & $1.754(2)$ & $\mathrm{C} 27-\mathrm{C} 35$ & $1.531(3)$ \\
\hline $\mathrm{S} 2-\mathrm{O} 4$ & $1.4427(15)$ & $\mathrm{C} 27-\mathrm{C} 28$ & $1.532(3)$ \\
\hline $\mathrm{S} 2-\mathrm{O} 6$ & $1.4478(13)$ & $\mathrm{C} 27-\mathrm{C} 26$ & $1.541(2)$ \\
\hline $\mathrm{S} 2-\mathrm{O} 5$ & $1.4491(14)$ & $\mathrm{C} 28-\mathrm{H} 28 \mathrm{~A}$ & 0.9700 \\
\hline $\mathrm{S} 2-\mathrm{C} 38$ & $1.748(2)$ & $\mathrm{C} 28-\mathrm{H} 28 \mathrm{~B}$ & 0.9700 \\
\hline $\mathrm{S} 3-\mathrm{O} 7$ & $1.4453(15)$ & $\mathrm{C} 32-\mathrm{C} 31$ & $1.528(2)$ \\
\hline $\mathrm{S} 3-\mathrm{O} 9$ & $1.4480(14)$ & $\mathrm{C} 32-\mathrm{H} 32 \mathrm{~A}$ & 0.9700 \\
\hline $\mathrm{S} 3-\mathrm{O} 8$ & $1.4511(14)$ & $\mathrm{C} 32-\mathrm{H} 32 \mathrm{~B}$ & 0.9700 \\
\hline $\mathrm{S} 3-\mathrm{C} 39$ & $1.751(2)$ & $\mathrm{C} 26-\mathrm{H} 26 \mathrm{~A}$ & 0.9700 \\
\hline $\mathrm{N} 2-\mathrm{C} 13$ & $1.4983(19)$ & $\mathrm{C} 26-\mathrm{H} 26 \mathrm{~B}$ & 0.9700 \\
\hline $\mathrm{N} 2-\mathrm{H} 2 \mathrm{~A}$ & $0.878(15)$ & $\mathrm{C} 15-\mathrm{C} 16$ & $1.531(3)$ \\
\hline $\mathrm{N} 2-\mathrm{H} 2 \mathrm{~B}$ & $0.873(15)$ & $\mathrm{C} 15-\mathrm{C} 22$ & $1.532(3)$ \\
\hline $\mathrm{N} 2-\mathrm{H} 2 \mathrm{C}$ & $0.896(15)$ & $\mathrm{C} 15-\mathrm{C} 23$ & $1.534(3)$ \\
\hline $\mathrm{N} 3-\mathrm{C} 25$ & $1.5026(19)$ & $\mathrm{C} 4-\mathrm{C} 5$ & $1.539(3)$ \\
\hline $\mathrm{N} 3-\mathrm{H} 3 \mathrm{~A}$ & $0.904(15)$ & $\mathrm{C} 4-\mathrm{H} 4 \mathrm{~A}$ & 0.9700 \\
\hline $\mathrm{N} 3-\mathrm{H} 3 \mathrm{~B}$ & $0.896(15)$ & $\mathrm{C} 4-\mathrm{H} 4 \mathrm{~B}$ & 0.9700 \\
\hline $\mathrm{N} 3-\mathrm{H} 3 \mathrm{C}$ & $0.890(15)$ & C16-H16A & 0.9700 \\
\hline $\mathrm{N} 1-\mathrm{C} 1$ & 1.5008 (19) & C16-H16B & 0.9700 \\
\hline $\mathrm{N} 1-\mathrm{H} 1 \mathrm{~A}$ & $0.901(15)$ & $\mathrm{C} 5-\mathrm{C} 12$ & $1.528(3)$ \\
\hline N1-H1B & $0.897(16)$ & $\mathrm{C} 5-\mathrm{C} 6$ & $1.535(3)$ \\
\hline $\mathrm{N} 1-\mathrm{H} 1 \mathrm{C}$ & $0.893(15)$ & $\mathrm{C} 31-\mathrm{C} 30$ & $1.520(3)$ \\
\hline $\mathrm{C} 1-\mathrm{C} 9$ & $1.524(2)$ & $\mathrm{C} 31-\mathrm{C} 34$ & $1.526(3)$ \\
\hline $\mathrm{C} 1-\mathrm{C} 2$ & $1.525(2)$ & C31-H31 & 0.9800 \\
\hline $\mathrm{C} 1-\mathrm{C} 8$ & $1.526(2)$ & $\mathrm{C} 34-\mathrm{H} 34 \mathrm{~A}$ & 0.9700 \\
\hline $\mathrm{C} 13-\mathrm{C} 21$ & $1.516(2)$ & $\mathrm{C} 34-\mathrm{H} 34 \mathrm{~B}$ & 0.9700 \\
\hline $\mathrm{C} 13-\mathrm{C} 20$ & $1.528(2)$ & $\mathrm{C} 18-\mathrm{C} 19$ & $1.519(3)$ \\
\hline $\mathrm{C} 13-\mathrm{C} 14$ & $1.529(2)$ & $\mathrm{C} 18-\mathrm{H} 18 \mathrm{~A}$ & 0.9700 \\
\hline $\mathrm{C} 21-\mathrm{C} 17$ & $1.540(2)$ & C18-H18B & 0.9700 \\
\hline $\mathrm{C} 21-\mathrm{H} 21 \mathrm{~A}$ & 0.9700 & $\mathrm{C} 30-\mathrm{H} 30 \mathrm{~A}$ & 0.9700 \\
\hline $\mathrm{C} 21-\mathrm{H} 21 \mathrm{~B}$ & 0.9700 & $\mathrm{C} 30-\mathrm{H} 30 \mathrm{~B}$ & 0.9700 \\
\hline $\mathrm{C} 2-\mathrm{C} 3$ & $1.532(2)$ & $\mathrm{C} 22-\mathrm{C} 19$ & $1.533(3)$ \\
\hline $\mathrm{C} 2-\mathrm{H} 2 \mathrm{D}$ & 0.9700 & $\mathrm{C} 22-\mathrm{H} 22 \mathrm{~A}$ & 0.9700 \\
\hline $\mathrm{C} 2-\mathrm{H} 2 \mathrm{E}$ & 0.9700 & $\mathrm{C} 22-\mathrm{H} 22 \mathrm{~B}$ & 0.9700 \\
\hline
\end{tabular}




\begin{tabular}{|c|c|c|c|}
\hline $\mathrm{C} 25-\mathrm{C} 33$ & $1.520(2)$ & $\mathrm{C} 11-\mathrm{H} 11 \mathrm{~A}$ & 0.9600 \\
\hline $\mathrm{C} 25-\mathrm{C} 26$ & $1.520(2)$ & $\mathrm{C} 11-\mathrm{H} 11 \mathrm{~B}$ & 0.9600 \\
\hline $\mathrm{C} 25-\mathrm{C} 32$ & $1.521(2)$ & $\mathrm{C} 11-\mathrm{H} 11 \mathrm{C}$ & 0.9600 \\
\hline $\mathrm{C} 8-\mathrm{C} 7$ & $1.529(2)$ & C6-H6A & 0.9700 \\
\hline $\mathrm{C} 8-\mathrm{H} 8 \mathrm{~A}$ & 0.9700 & C6-H6B & 0.9700 \\
\hline $\mathrm{C} 8-\mathrm{H} 8 \mathrm{~B}$ & 0.9700 & C19-H19 & 0.9800 \\
\hline $\mathrm{C} 14-\mathrm{C} 15$ & $1.538(2)$ & C37-H37A & 0.9600 \\
\hline $\mathrm{C} 14-\mathrm{H} 14 \mathrm{~A}$ & 0.9700 & C37-H37B & 0.9600 \\
\hline C14-H14B & 0.9700 & C37-H37C & 0.9600 \\
\hline $\mathrm{C} 33-\mathrm{C} 29$ & $1.535(2)$ & $\mathrm{C} 24-\mathrm{H} 24 \mathrm{~A}$ & 0.9600 \\
\hline $\mathrm{C} 33-\mathrm{H} 33 \mathrm{~A}$ & 0.9700 & $\mathrm{C} 24-\mathrm{H} 24 \mathrm{~B}$ & 0.9600 \\
\hline C $33-\mathrm{H} 33 \mathrm{~B}$ & 0.9700 & $\mathrm{C} 24-\mathrm{H} 24 \mathrm{C}$ & 0.9600 \\
\hline $\mathrm{C} 3-\mathrm{C} 4$ & $1.533(3)$ & C39-H39A & 0.9600 \\
\hline $\mathrm{C} 3-\mathrm{C} 11$ & $1.533(2)$ & C39-H39B & 0.9600 \\
\hline $\mathrm{C} 3-\mathrm{C} 10$ & $1.533(2)$ & $\mathrm{C} 39-\mathrm{H} 39 \mathrm{C}$ & 0.9600 \\
\hline $\mathrm{C} 9-\mathrm{C} 5$ & $1.534(2)$ & $\mathrm{C} 23-\mathrm{H} 23 \mathrm{~A}$ & 0.9600 \\
\hline $\mathrm{C} 9-\mathrm{H} 9 \mathrm{~A}$ & 0.9700 & $\mathrm{C} 23-\mathrm{H} 23 \mathrm{~B}$ & 0.9600 \\
\hline C9-H9B & 0.9700 & $\mathrm{C} 23-\mathrm{H} 23 \mathrm{C}$ & 0.9600 \\
\hline $\mathrm{C} 29-\mathrm{C} 36$ & $1.527(3)$ & $\mathrm{C} 36-\mathrm{H} 36 \mathrm{~A}$ & 0.9600 \\
\hline $\mathrm{C} 29-\mathrm{C} 30$ & $1.532(3)$ & C36-H36B & 0.9600 \\
\hline $\mathrm{C} 29-\mathrm{C} 28$ & $1.533(2)$ & $\mathrm{C} 36-\mathrm{H} 36 \mathrm{C}$ & 0.9600 \\
\hline $\mathrm{C} 17-\mathrm{C} 24$ & $1.524(3)$ & C $38-\mathrm{H} 38 \mathrm{~A}$ & 0.9600 \\
\hline $\mathrm{C} 17-\mathrm{C} 16$ & $1.530(2)$ & C $38-\mathrm{H} 38 \mathrm{~B}$ & 0.9600 \\
\hline $\mathrm{C} 17-\mathrm{C} 18$ & $1.536(3)$ & $\mathrm{C} 38-\mathrm{H} 38 \mathrm{C}$ & 0.9600 \\
\hline $\mathrm{C} 20-\mathrm{C} 19$ & $1.532(3)$ & C35-H35A & 0.9600 \\
\hline $\mathrm{C} 20-\mathrm{H} 20 \mathrm{~A}$ & 0.9700 & C $35-\mathrm{H} 35 \mathrm{~B}$ & 0.9600 \\
\hline $\mathrm{C} 20-\mathrm{H} 20 \mathrm{~B}$ & 0.9700 & $\mathrm{C} 35-\mathrm{H} 35 \mathrm{C}$ & 0.9600 \\
\hline $\mathrm{C} 7-\mathrm{C} 6$ & $1.520(3)$ & $\mathrm{C} 12-\mathrm{H} 12 \mathrm{~A}$ & 0.9600 \\
\hline $\mathrm{C} 7-\mathrm{C} 10$ & $1.530(3)$ & $\mathrm{C} 12-\mathrm{H} 12 \mathrm{~B}$ & 0.9600 \\
\hline C7-H7 & 0.9800 & $\mathrm{C} 12-\mathrm{H} 12 \mathrm{C}$ & 0.9600 \\
\hline $\mathrm{O} 3-\mathrm{S} 1-\mathrm{O} 2$ & $112.16(8)$ & $\mathrm{C} 27-\mathrm{C} 28-\mathrm{H} 28 \mathrm{~B}$ & 109.3 \\
\hline $\mathrm{O} 3-\mathrm{S} 1-\mathrm{O} 1$ & $112.49(9)$ & $\mathrm{C} 29-\mathrm{C} 28-\mathrm{H} 28 \mathrm{~B}$ & 109.3 \\
\hline $\mathrm{O} 2-\mathrm{S} 1-\mathrm{O} 1$ & $112.24(9)$ & $\mathrm{H} 28 \mathrm{~A}-\mathrm{C} 28-\mathrm{H} 28 \mathrm{~B}$ & 107.9 \\
\hline $\mathrm{O} 3-\mathrm{S} 1-\mathrm{C} 37$ & $106.48(10)$ & $\mathrm{C} 25-\mathrm{C} 32-\mathrm{C} 31$ & $108.64(14)$ \\
\hline $\mathrm{O} 2-\mathrm{S} 1-\mathrm{C} 37$ & $106.90(11)$ & $\mathrm{C} 25-\mathrm{C} 32-\mathrm{H} 32 \mathrm{~A}$ & 110.0 \\
\hline $\mathrm{O} 1-\mathrm{S} 1-\mathrm{C} 37$ & $106.03(11)$ & $\mathrm{C} 31-\mathrm{C} 32-\mathrm{H} 32 \mathrm{~A}$ & 110.0 \\
\hline $\mathrm{O} 4-\mathrm{S} 2-\mathrm{O} 6$ & $112.33(9)$ & $\mathrm{C} 25-\mathrm{C} 32-\mathrm{H} 32 \mathrm{~B}$ & 110.0 \\
\hline $\mathrm{O} 4-\mathrm{S} 2-\mathrm{O} 5$ & $112.53(10)$ & $\mathrm{C} 31-\mathrm{C} 32-\mathrm{H} 32 \mathrm{~B}$ & 110.0 \\
\hline $\mathrm{O} 6-\mathrm{S} 2-\mathrm{O} 5$ & $111.99(8)$ & $\mathrm{H} 32 \mathrm{~A}-\mathrm{C} 32-\mathrm{H} 32 \mathrm{~B}$ & 108.3 \\
\hline $\mathrm{O} 4-\mathrm{S} 2-\mathrm{C} 38$ & $106.23(12)$ & $\mathrm{C} 25-\mathrm{C} 26-\mathrm{C} 27$ & $109.43(13)$ \\
\hline $\mathrm{O} 6-\mathrm{S} 2-\mathrm{C} 38$ & $107.04(11)$ & $\mathrm{C} 25-\mathrm{C} 26-\mathrm{H} 26 \mathrm{~A}$ & 109.8 \\
\hline $\mathrm{O} 5-\mathrm{S} 2-\mathrm{C} 38$ & $106.18(11)$ & $\mathrm{C} 27-\mathrm{C} 26-\mathrm{H} 26 \mathrm{~A}$ & 109.8 \\
\hline $\mathrm{O} 7-\mathrm{S} 3-\mathrm{O} 9$ & $112.24(10)$ & $\mathrm{C} 25-\mathrm{C} 26-\mathrm{H} 26 \mathrm{~B}$ & 109.8 \\
\hline $\mathrm{O} 7-\mathrm{S} 3-\mathrm{O} 8$ & $112.80(10)$ & $\mathrm{C} 27-\mathrm{C} 26-\mathrm{H} 26 \mathrm{~B}$ & 109.8 \\
\hline $\mathrm{O} 9-\mathrm{S} 3-\mathrm{O} 8$ & $111.98(8)$ & $\mathrm{H} 26 \mathrm{~A}-\mathrm{C} 26-\mathrm{H} 26 \mathrm{~B}$ & 108.2 \\
\hline $\mathrm{O} 7-\mathrm{S} 3-\mathrm{C} 39$ & $106.65(12)$ & $\mathrm{C} 16-\mathrm{C} 15-\mathrm{C} 22$ & $108.80(16)$ \\
\hline $\mathrm{O} 9-\mathrm{S} 3-\mathrm{C} 39$ & $105.98(11)$ & $\mathrm{C} 16-\mathrm{C} 15-\mathrm{C} 23$ & $110.50(17)$ \\
\hline
\end{tabular}




\begin{tabular}{|c|c|c|c|}
\hline $\mathrm{O} 8-\mathrm{S} 3-\mathrm{C} 39$ & $106.65(11)$ & $\mathrm{C} 22-\mathrm{C} 15-\mathrm{C} 23$ & $111.15(18)$ \\
\hline $\mathrm{C} 13-\mathrm{N} 2-\mathrm{H} 2 \mathrm{~A}$ & $108.9(13)$ & $\mathrm{C} 16-\mathrm{C} 15-\mathrm{C} 14$ & $108.38(14)$ \\
\hline $\mathrm{C} 13-\mathrm{N} 2-\mathrm{H} 2 \mathrm{~B}$ & $108.9(13)$ & $\mathrm{C} 22-\mathrm{C} 15-\mathrm{C} 14$ & $108.47(15)$ \\
\hline $\mathrm{H} 2 \mathrm{~A}-\mathrm{N} 2-\mathrm{H} 2 \mathrm{~B}$ & $108.3(18)$ & $\mathrm{C} 23-\mathrm{C} 15-\mathrm{C} 14$ & $109.49(16)$ \\
\hline $\mathrm{C} 13-\mathrm{N} 2-\mathrm{H} 2 \mathrm{C}$ & $107.2(13)$ & $\mathrm{C} 3-\mathrm{C} 4-\mathrm{C} 5$ & $111.38(15)$ \\
\hline $\mathrm{H} 2 \mathrm{~A}-\mathrm{N} 2-\mathrm{H} 2 \mathrm{C}$ & $109.0(18)$ & $\mathrm{C} 3-\mathrm{C} 4-\mathrm{H} 4 \mathrm{~A}$ & 109.4 \\
\hline $\mathrm{H} 2 \mathrm{~B}-\mathrm{N} 2-\mathrm{H} 2 \mathrm{C}$ & $114.4(18)$ & $\mathrm{C} 5-\mathrm{C} 4-\mathrm{H} 4 \mathrm{~A}$ & 109.4 \\
\hline $\mathrm{C} 25-\mathrm{N} 3-\mathrm{H} 3 \mathrm{~A}$ & $111.5(13)$ & $\mathrm{C} 3-\mathrm{C} 4-\mathrm{H} 4 \mathrm{~B}$ & 109.4 \\
\hline $\mathrm{C} 25-\mathrm{N} 3-\mathrm{H} 3 \mathrm{~B}$ & $111.5(13)$ & $\mathrm{C} 5-\mathrm{C} 4-\mathrm{H} 4 \mathrm{~B}$ & 109.4 \\
\hline $\mathrm{H} 3 \mathrm{~A}-\mathrm{N} 3-\mathrm{H} 3 \mathrm{~B}$ & $105.7(18)$ & $\mathrm{H} 4 \mathrm{~A}-\mathrm{C} 4-\mathrm{H} 4 \mathrm{~B}$ & 108.0 \\
\hline $\mathrm{C} 25-\mathrm{N} 3-\mathrm{H} 3 \mathrm{C}$ & $111.2(13)$ & $\mathrm{C} 17-\mathrm{C} 16-\mathrm{C} 15$ & $111.81(15)$ \\
\hline $\mathrm{H} 3 \mathrm{~A}-\mathrm{N} 3-\mathrm{H} 3 \mathrm{C}$ & $105.8(18)$ & $\mathrm{C} 17-\mathrm{C} 16-\mathrm{H} 16 \mathrm{~A}$ & 109.3 \\
\hline $\mathrm{H} 3 \mathrm{~B}-\mathrm{N} 3-\mathrm{H} 3 \mathrm{C}$ & $110.8(19)$ & $\mathrm{C} 15-\mathrm{C} 16-\mathrm{H} 16 \mathrm{~A}$ & 109.3 \\
\hline $\mathrm{C} 1-\mathrm{N} 1-\mathrm{H} 1 \mathrm{~A}$ & $106.7(14)$ & $\mathrm{C} 17-\mathrm{C} 16-\mathrm{H} 16 \mathrm{~B}$ & 109.3 \\
\hline $\mathrm{C} 1-\mathrm{N} 1-\mathrm{H} 1 \mathrm{~B}$ & $107.8(14)$ & $\mathrm{C} 15-\mathrm{C} 16-\mathrm{H} 16 \mathrm{~B}$ & 109.3 \\
\hline $\mathrm{H} 1 \mathrm{~A}-\mathrm{N} 1-\mathrm{H} 1 \mathrm{~B}$ & $113(2)$ & $\mathrm{H} 16 \mathrm{~A}-\mathrm{C} 16-\mathrm{H} 16 \mathrm{~B}$ & 107.9 \\
\hline $\mathrm{C} 1-\mathrm{N} 1-\mathrm{H} 1 \mathrm{C}$ & $107.3(14)$ & $\mathrm{C} 12-\mathrm{C} 5-\mathrm{C} 9$ & $109.66(18)$ \\
\hline $\mathrm{H} 1 \mathrm{~A}-\mathrm{N} 1-\mathrm{H} 1 \mathrm{C}$ & 112.9 (19) & $\mathrm{C} 12-\mathrm{C} 5-\mathrm{C} 6$ & $110.9(2)$ \\
\hline $\mathrm{H} 1 \mathrm{~B}-\mathrm{N} 1-\mathrm{H} 1 \mathrm{C}$ & $109(2)$ & $\mathrm{C} 9-\mathrm{C} 5-\mathrm{C} 6$ & $108.75(16)$ \\
\hline $\mathrm{N} 1-\mathrm{C} 1-\mathrm{C} 9$ & $108.80(13)$ & $\mathrm{C} 12-\mathrm{C} 5-\mathrm{C} 4$ & $110.77(18)$ \\
\hline $\mathrm{N} 1-\mathrm{C} 1-\mathrm{C} 2$ & $109.46(12)$ & $\mathrm{C} 9-\mathrm{C} 5-\mathrm{C} 4$ & $108.30(15)$ \\
\hline $\mathrm{C} 9-\mathrm{C} 1-\mathrm{C} 2$ & $110.08(13)$ & $\mathrm{C} 6-\mathrm{C} 5-\mathrm{C} 4$ & $108.38(16)$ \\
\hline $\mathrm{N} 1-\mathrm{C} 1-\mathrm{C} 8$ & $108.44(13)$ & $\mathrm{C} 30-\mathrm{C} 31-\mathrm{C} 34$ & $109.47(17)$ \\
\hline $\mathrm{C} 9-\mathrm{C} 1-\mathrm{C} 8$ & $110.26(14)$ & $\mathrm{C} 30-\mathrm{C} 31-\mathrm{C} 32$ & $110.15(16)$ \\
\hline $\mathrm{C} 2-\mathrm{C} 1-\mathrm{C} 8$ & $109.76(13)$ & $\mathrm{C} 34-\mathrm{C} 31-\mathrm{C} 32$ & $108.73(16)$ \\
\hline $\mathrm{N} 2-\mathrm{C} 13-\mathrm{C} 21$ & $109.02(12)$ & $\mathrm{C} 30-\mathrm{C} 31-\mathrm{H} 31$ & 109.5 \\
\hline $\mathrm{N} 2-\mathrm{C} 13-\mathrm{C} 20$ & $108.75(13)$ & C34-C31-H31 & 109.5 \\
\hline $\mathrm{C} 21-\mathrm{C} 13-\mathrm{C} 20$ & $110.00(14)$ & $\mathrm{C} 32-\mathrm{C} 31-\mathrm{H} 31$ & 109.5 \\
\hline $\mathrm{N} 2-\mathrm{C} 13-\mathrm{C} 14$ & $108.56(12)$ & $\mathrm{C} 31-\mathrm{C} 34-\mathrm{C} 27$ & $110.87(15)$ \\
\hline $\mathrm{C} 21-\mathrm{C} 13-\mathrm{C} 14$ & $110.25(13)$ & C31-C34-H34A & 109.5 \\
\hline $\mathrm{C} 20-\mathrm{C} 13-\mathrm{C} 14$ & $110.23(14)$ & $\mathrm{C} 27-\mathrm{C} 34-\mathrm{H} 34 \mathrm{~A}$ & 109.5 \\
\hline $\mathrm{C} 13-\mathrm{C} 21-\mathrm{C} 17$ & $109.86(13)$ & C31-C34-H34B & 109.5 \\
\hline $\mathrm{C} 13-\mathrm{C} 21-\mathrm{H} 21 \mathrm{~A}$ & 109.7 & $\mathrm{C} 27-\mathrm{C} 34-\mathrm{H} 34 \mathrm{~B}$ & 109.5 \\
\hline $\mathrm{C} 17-\mathrm{C} 21-\mathrm{H} 21 \mathrm{~A}$ & 109.7 & $\mathrm{H} 34 \mathrm{~A}-\mathrm{C} 34-\mathrm{H} 34 \mathrm{~B}$ & 108.1 \\
\hline $\mathrm{C} 13-\mathrm{C} 21-\mathrm{H} 21 \mathrm{~B}$ & 109.7 & $\mathrm{C} 19-\mathrm{C} 18-\mathrm{C} 17$ & $110.38(15)$ \\
\hline $\mathrm{C} 17-\mathrm{C} 21-\mathrm{H} 21 \mathrm{~B}$ & 109.7 & $\mathrm{C} 19-\mathrm{C} 18-\mathrm{H} 18 \mathrm{~A}$ & 109.6 \\
\hline $\mathrm{H} 21 \mathrm{~A}-\mathrm{C} 21-\mathrm{H} 21 \mathrm{~B}$ & 108.2 & $\mathrm{C} 17-\mathrm{C} 18-\mathrm{H} 18 \mathrm{~A}$ & 109.6 \\
\hline $\mathrm{C} 1-\mathrm{C} 2-\mathrm{C} 3$ & $110.01(12)$ & $\mathrm{C} 19-\mathrm{C} 18-\mathrm{H} 18 \mathrm{~B}$ & 109.6 \\
\hline $\mathrm{C} 1-\mathrm{C} 2-\mathrm{H} 2 \mathrm{D}$ & 109.7 & $\mathrm{C} 17-\mathrm{C} 18-\mathrm{H} 18 \mathrm{~B}$ & 109.6 \\
\hline $\mathrm{C} 3-\mathrm{C} 2-\mathrm{H} 2 \mathrm{D}$ & 109.7 & $\mathrm{H} 18 \mathrm{~A}-\mathrm{C} 18-\mathrm{H} 18 \mathrm{~B}$ & 108.1 \\
\hline $\mathrm{C} 1-\mathrm{C} 2-\mathrm{H} 2 \mathrm{E}$ & 109.7 & $\mathrm{C} 31-\mathrm{C} 30-\mathrm{C} 29$ & $110.54(15)$ \\
\hline $\mathrm{C} 3-\mathrm{C} 2-\mathrm{H} 2 \mathrm{E}$ & 109.7 & $\mathrm{C} 31-\mathrm{C} 30-\mathrm{H} 30 \mathrm{~A}$ & 109.5 \\
\hline $\mathrm{H} 2 \mathrm{D}-\mathrm{C} 2-\mathrm{H} 2 \mathrm{E}$ & 108.2 & $\mathrm{C} 29-\mathrm{C} 30-\mathrm{H} 30 \mathrm{~A}$ & 109.5 \\
\hline $\mathrm{N} 3-\mathrm{C} 25-\mathrm{C} 33$ & $109.30(12)$ & $\mathrm{C} 31-\mathrm{C} 30-\mathrm{H} 30 \mathrm{~B}$ & 109.5 \\
\hline $\mathrm{N} 3-\mathrm{C} 25-\mathrm{C} 26$ & $108.86(12)$ & С29- $\mathrm{C} 30-\mathrm{H} 30 \mathrm{~B}$ & 109.5 \\
\hline $\mathrm{C} 33-\mathrm{C} 25-\mathrm{C} 26$ & $109.96(13)$ & $\mathrm{H} 30 \mathrm{~A}-\mathrm{C} 30-\mathrm{H} 30 \mathrm{~B}$ & 108.1 \\
\hline $\mathrm{N} 3-\mathrm{C} 25-\mathrm{C} 32$ & $108.23(13)$ & $\mathrm{C} 15-\mathrm{C} 22-\mathrm{C} 19$ & $110.50(15)$ \\
\hline $\mathrm{C} 33-\mathrm{C} 25-\mathrm{C} 32$ & $110.02(13)$ & $\mathrm{C} 15-\mathrm{C} 22-\mathrm{H} 22 \mathrm{~A}$ & 109.5 \\
\hline
\end{tabular}




\begin{tabular}{|c|c|}
\hline $\mathrm{C} 26-\mathrm{C} 25-\mathrm{C} 32$ & $110.44(14)$ \\
\hline $\mathrm{C} 1-\mathrm{C} 8-\mathrm{C} 7$ & $108.38(14)$ \\
\hline $\mathrm{C} 1-\mathrm{C} 8-\mathrm{H} 8 \mathrm{~A}$ & 110.0 \\
\hline $\mathrm{C} 7-\mathrm{C} 8-\mathrm{H} 8 \mathrm{~A}$ & 110.0 \\
\hline $\mathrm{C} 1-\mathrm{C} 8-\mathrm{H} 8 \mathrm{~B}$ & 110.0 \\
\hline $\mathrm{C} 7-\mathrm{C} 8-\mathrm{H} 8 \mathrm{~B}$ & 110.0 \\
\hline $\mathrm{H} 8 \mathrm{~A}-\mathrm{C} 8-\mathrm{H} 8 \mathrm{~B}$ & 108.4 \\
\hline $\mathrm{C} 13-\mathrm{C} 14-\mathrm{C} 15$ & $109.31(13)$ \\
\hline $\mathrm{C} 13-\mathrm{C} 14-\mathrm{H} 14 \mathrm{~A}$ & 109.8 \\
\hline $\mathrm{C} 15-\mathrm{C} 14-\mathrm{H} 14 \mathrm{~A}$ & 109.8 \\
\hline $\mathrm{C} 13-\mathrm{C} 14-\mathrm{H} 14 \mathrm{~B}$ & 109.8 \\
\hline $\mathrm{C} 15-\mathrm{C} 14-\mathrm{H} 14 \mathrm{~B}$ & 109.8 \\
\hline $\mathrm{H} 14 \mathrm{~A}-\mathrm{C} 14-\mathrm{H} 14 \mathrm{~B}$ & 108.3 \\
\hline $\mathrm{C} 25-\mathrm{C} 33-\mathrm{C} 29$ & $110.04(13)$ \\
\hline $\mathrm{C} 25-\mathrm{C} 33-\mathrm{H} 33 \mathrm{~A}$ & 109.7 \\
\hline $\mathrm{C} 29-\mathrm{C} 33-\mathrm{H} 33 \mathrm{~A}$ & 109.7 \\
\hline $\mathrm{C} 25-\mathrm{C} 33-\mathrm{H} 33 \mathrm{~B}$ & 109.7 \\
\hline С29-C $33-\mathrm{H} 33 \mathrm{~B}$ & 109.7 \\
\hline $\mathrm{H} 33 \mathrm{~A}-\mathrm{C} 33-\mathrm{H} 33 \mathrm{~B}$ & 108.2 \\
\hline $\mathrm{C} 2-\mathrm{C} 3-\mathrm{C} 4$ & $108.56(13)$ \\
\hline $\mathrm{C} 2-\mathrm{C} 3-\mathrm{C} 11$ & $110.40(14)$ \\
\hline $\mathrm{C} 4-\mathrm{C} 3-\mathrm{C} 11$ & $110.30(15)$ \\
\hline $\mathrm{C} 2-\mathrm{C} 3-\mathrm{C} 10$ & $108.42(14)$ \\
\hline $\mathrm{C} 4-\mathrm{C} 3-\mathrm{C} 10$ & $108.78(15)$ \\
\hline $\mathrm{C} 11-\mathrm{C} 3-\mathrm{C} 10$ & $110.32(14)$ \\
\hline $\mathrm{C} 1-\mathrm{C} 9-\mathrm{C} 5$ & $109.68(14)$ \\
\hline $\mathrm{C} 1-\mathrm{C} 9-\mathrm{H} 9 \mathrm{~A}$ & 109.7 \\
\hline $\mathrm{C} 5-\mathrm{C} 9-\mathrm{H} 9 \mathrm{~A}$ & 109.7 \\
\hline $\mathrm{C} 1-\mathrm{C} 9-\mathrm{H} 9 \mathrm{~B}$ & 109.7 \\
\hline $\mathrm{C} 5-\mathrm{C} 9-\mathrm{H} 9 \mathrm{~B}$ & 109.7 \\
\hline $\mathrm{H} 9 \mathrm{~A}-\mathrm{C} 9-\mathrm{H} 9 \mathrm{~B}$ & 108.2 \\
\hline $\mathrm{C} 36-\mathrm{C} 29-\mathrm{C} 30$ & $111.16(18)$ \\
\hline $\mathrm{C} 36-\mathrm{C} 29-\mathrm{C} 28$ & $110.63(17)$ \\
\hline $\mathrm{C} 30-\mathrm{C} 29-\mathrm{C} 28$ & $108.68(16)$ \\
\hline $\mathrm{C} 36-\mathrm{C} 29-\mathrm{C} 33$ & $109.98(16)$ \\
\hline $\mathrm{C} 30-\mathrm{C} 29-\mathrm{C} 33$ & $107.91(15)$ \\
\hline $\mathrm{C} 28-\mathrm{C} 29-\mathrm{C} 33$ & $108.40(14)$ \\
\hline $\mathrm{C} 24-\mathrm{C} 17-\mathrm{C} 16$ & $110.87(17)$ \\
\hline $\mathrm{C} 24-\mathrm{C} 17-\mathrm{C} 18$ & $110.44(17)$ \\
\hline $\mathrm{C} 16-\mathrm{C} 17-\mathrm{C} 18$ & $108.76(15)$ \\
\hline $\mathrm{C} 24-\mathrm{C} 17-\mathrm{C} 21$ & $110.12(15)$ \\
\hline $\mathrm{C} 16-\mathrm{C} 17-\mathrm{C} 21$ & $108.09(14)$ \\
\hline $\mathrm{C} 18-\mathrm{C} 17-\mathrm{C} 21$ & $108.49(15)$ \\
\hline $\mathrm{C} 13-\mathrm{C} 20-\mathrm{C} 19$ & $108.48(14)$ \\
\hline $\mathrm{C} 13-\mathrm{C} 20-\mathrm{H} 20 \mathrm{~A}$ & 110.0 \\
\hline $\mathrm{C} 19-\mathrm{C} 20-\mathrm{H} 20 \mathrm{~A}$ & 110.0 \\
\hline $\mathrm{C} 13-\mathrm{C} 20-\mathrm{H} 20 \mathrm{~B}$ & 110.0 \\
\hline $\mathrm{C} 19-\mathrm{C} 20-\mathrm{H} 20 \mathrm{~B}$ & \\
\hline
\end{tabular}

\begin{tabular}{|c|c|}
\hline $\mathrm{C} 19-\mathrm{C} 22-\mathrm{H} 22 \mathrm{~A}$ & 109.5 \\
\hline $\mathrm{C} 15-\mathrm{C} 22-\mathrm{H} 22 \mathrm{~B}$ & 109.5 \\
\hline $\mathrm{C} 19-\mathrm{C} 22-\mathrm{H} 22 \mathrm{~B}$ & 109.5 \\
\hline $\mathrm{H} 22 \mathrm{~A}-\mathrm{C} 22-\mathrm{H} 22 \mathrm{~B}$ & 108.1 \\
\hline $\mathrm{C} 3-\mathrm{C} 11-\mathrm{H} 11 \mathrm{~A}$ & 109.5 \\
\hline $\mathrm{C} 3-\mathrm{C} 11-\mathrm{H} 11 \mathrm{~B}$ & 109.5 \\
\hline $\mathrm{H} 11 \mathrm{~A}-\mathrm{C} 11-\mathrm{H} 11 \mathrm{~B}$ & 109.5 \\
\hline $\mathrm{C} 3-\mathrm{C} 11-\mathrm{H} 11 \mathrm{C}$ & 109.5 \\
\hline $\mathrm{H} 11 \mathrm{~A}-\mathrm{C} 11-\mathrm{H} 11 \mathrm{C}$ & 109.5 \\
\hline $\mathrm{H} 11 \mathrm{~B}-\mathrm{C} 11-\mathrm{H} 11 \mathrm{C}$ & 109.5 \\
\hline $\mathrm{C} 7-\mathrm{C} 6-\mathrm{C} 5$ & $110.54(14)$ \\
\hline $\mathrm{C} 7-\mathrm{C} 6-\mathrm{H} 6 \mathrm{~A}$ & 109.5 \\
\hline $\mathrm{C} 5-\mathrm{C} 6-\mathrm{H} 6 \mathrm{~A}$ & 109.5 \\
\hline $\mathrm{C} 7-\mathrm{C} 6-\mathrm{H} 6 \mathrm{~B}$ & 109.5 \\
\hline $\mathrm{C} 5-\mathrm{C} 6-\mathrm{H} 6 \mathrm{~B}$ & 109.5 \\
\hline $\mathrm{H} 6 \mathrm{~A}-\mathrm{C} 6-\mathrm{H} 6 \mathrm{~B}$ & 108.1 \\
\hline $\mathrm{C} 18-\mathrm{C} 19-\mathrm{C} 20$ & $109.84(16)$ \\
\hline $\mathrm{C} 18-\mathrm{C} 19-\mathrm{C} 22$ & $109.75(18)$ \\
\hline $\mathrm{C} 20-\mathrm{C} 19-\mathrm{C} 22$ & $108.95(17)$ \\
\hline $\mathrm{C} 18-\mathrm{C} 19-\mathrm{H} 19$ & 109.4 \\
\hline $\mathrm{C} 20-\mathrm{C} 19-\mathrm{H} 19$ & 109.4 \\
\hline $\mathrm{C} 22-\mathrm{C} 19-\mathrm{H} 19$ & 109.4 \\
\hline $\mathrm{S} 1-\mathrm{C} 37-\mathrm{H} 37 \mathrm{~A}$ & 109.5 \\
\hline $\mathrm{S} 1-\mathrm{C} 37-\mathrm{H} 37 \mathrm{~B}$ & 109.5 \\
\hline $\mathrm{H} 37 \mathrm{~A}-\mathrm{C} 37-\mathrm{H} 37 \mathrm{~B}$ & 109.5 \\
\hline $\mathrm{S} 1-\mathrm{C} 37-\mathrm{H} 37 \mathrm{C}$ & 109.5 \\
\hline $\mathrm{H} 37 \mathrm{~A}-\mathrm{C} 37-\mathrm{H} 37 \mathrm{C}$ & 109.5 \\
\hline $\mathrm{H} 37 \mathrm{~B}-\mathrm{C} 37-\mathrm{H} 37 \mathrm{C}$ & 109.5 \\
\hline $\mathrm{C} 17-\mathrm{C} 24-\mathrm{H} 24 \mathrm{~A}$ & 109.5 \\
\hline $\mathrm{C} 17-\mathrm{C} 24-\mathrm{H} 24 \mathrm{~B}$ & 109.5 \\
\hline $\mathrm{H} 24 \mathrm{~A}-\mathrm{C} 24-\mathrm{H} 24 \mathrm{~B}$ & 109.5 \\
\hline $\mathrm{C} 17-\mathrm{C} 24-\mathrm{H} 24 \mathrm{C}$ & 109.5 \\
\hline $\mathrm{H} 24 \mathrm{~A}-\mathrm{C} 24-\mathrm{H} 24 \mathrm{C}$ & 109.5 \\
\hline $\mathrm{H} 24 \mathrm{~B}-\mathrm{C} 24-\mathrm{H} 24 \mathrm{C}$ & 109.5 \\
\hline $\mathrm{S} 3-\mathrm{C} 39-\mathrm{H} 39 \mathrm{~A}$ & 109.5 \\
\hline $\mathrm{S} 3-\mathrm{C} 39-\mathrm{H} 39 \mathrm{~B}$ & 109.5 \\
\hline $\mathrm{H} 39 \mathrm{~A}-\mathrm{C} 39-\mathrm{H} 39 \mathrm{~B}$ & 109.5 \\
\hline $\mathrm{S} 3-\mathrm{C} 39-\mathrm{H} 39 \mathrm{C}$ & 109.5 \\
\hline H39A-C39-H39C & 109.5 \\
\hline $\mathrm{H} 39 \mathrm{~B}-\mathrm{C} 39-\mathrm{H} 39 \mathrm{C}$ & 109.5 \\
\hline $\mathrm{C} 15-\mathrm{C} 23-\mathrm{H} 23 \mathrm{~A}$ & 109.5 \\
\hline $\mathrm{C} 15-\mathrm{C} 23-\mathrm{H} 23 \mathrm{~B}$ & 109.5 \\
\hline $\mathrm{H} 23 \mathrm{~A}-\mathrm{C} 23-\mathrm{H} 23 \mathrm{~B}$ & 109.5 \\
\hline $\mathrm{C} 15-\mathrm{C} 23-\mathrm{H} 23 \mathrm{C}$ & 109.5 \\
\hline $\mathrm{H} 23 \mathrm{~A}-\mathrm{C} 23-\mathrm{H} 23 \mathrm{C}$ & 109.5 \\
\hline $\mathrm{H} 23 \mathrm{~B}-\mathrm{C} 23-\mathrm{H} 23 \mathrm{C}$ & 109.5 \\
\hline $\mathrm{C} 29-\mathrm{C} 36-\mathrm{H} 36 \mathrm{~A}$ & 109.5 \\
\hline $\mathrm{C} 29-\mathrm{C} 36-\mathrm{H} 36 \mathrm{~B}$ & 109.5 \\
\hline
\end{tabular}




\begin{tabular}{|c|c|c|c|}
\hline $\mathrm{H} 20 \mathrm{~A}-\mathrm{C} 20-\mathrm{H} 20 \mathrm{~B}$ & 108.4 & $\mathrm{H} 36 \mathrm{~A}-\mathrm{C} 36-\mathrm{H} 36 \mathrm{~B}$ & 109.5 \\
\hline $\mathrm{C} 6-\mathrm{C} 7-\mathrm{C} 8$ & $109.66(16)$ & $\mathrm{C} 29-\mathrm{C} 36-\mathrm{H} 36 \mathrm{C}$ & 109.5 \\
\hline $\mathrm{C} 6-\mathrm{C} 7-\mathrm{C} 10$ & $109.48(17)$ & $\mathrm{H} 36 \mathrm{~A}-\mathrm{C} 36-\mathrm{H} 36 \mathrm{C}$ & 109.5 \\
\hline $\mathrm{C} 8-\mathrm{C} 7-\mathrm{C} 10$ & $109.83(15)$ & $\mathrm{H} 36 \mathrm{~B}-\mathrm{C} 36-\mathrm{H} 36 \mathrm{C}$ & 109.5 \\
\hline $\mathrm{C} 6-\mathrm{C} 7-\mathrm{H} 7$ & 109.3 & $\mathrm{~S} 2-\mathrm{C} 38-\mathrm{H} 38 \mathrm{~A}$ & 109.5 \\
\hline $\mathrm{C} 8-\mathrm{C} 7-\mathrm{H} 7$ & 109.3 & $\mathrm{~S} 2-\mathrm{C} 38-\mathrm{H} 38 \mathrm{~B}$ & 109.5 \\
\hline $\mathrm{C} 10-\mathrm{C} 7-\mathrm{H} 7$ & 109.3 & $\mathrm{H} 38 \mathrm{~A}-\mathrm{C} 38-\mathrm{H} 38 \mathrm{~B}$ & 109.5 \\
\hline $\mathrm{C} 7-\mathrm{C} 10-\mathrm{C} 3$ & $110.09(14)$ & $\mathrm{S} 2-\mathrm{C} 38-\mathrm{H} 38 \mathrm{C}$ & 109.5 \\
\hline $\mathrm{C} 7-\mathrm{C} 10-\mathrm{H} 10 \mathrm{~A}$ & 109.6 & $\mathrm{H} 38 \mathrm{~A}-\mathrm{C} 38-\mathrm{H} 38 \mathrm{C}$ & 109.5 \\
\hline $\mathrm{C} 3-\mathrm{C} 10-\mathrm{H} 10 \mathrm{~A}$ & 109.6 & $\mathrm{H} 38 \mathrm{~B}-\mathrm{C} 38-\mathrm{H} 38 \mathrm{C}$ & 109.5 \\
\hline $\mathrm{C} 7-\mathrm{C} 10-\mathrm{H} 10 \mathrm{~B}$ & 109.6 & $\mathrm{C} 27-\mathrm{C} 35-\mathrm{H} 35 \mathrm{~A}$ & 109.5 \\
\hline $\mathrm{C} 3-\mathrm{C} 10-\mathrm{H} 10 \mathrm{~B}$ & 109.6 & $\mathrm{C} 27-\mathrm{C} 35-\mathrm{H} 35 \mathrm{~B}$ & 109.5 \\
\hline $\mathrm{H} 10 \mathrm{~A}-\mathrm{C} 10-\mathrm{H} 10 \mathrm{~B}$ & 108.2 & $\mathrm{H} 35 \mathrm{~A}-\mathrm{C} 35-\mathrm{H} 35 \mathrm{~B}$ & 109.5 \\
\hline C34-C27-C35 & $111.57(19)$ & $\mathrm{C} 27-\mathrm{C} 35-\mathrm{H} 35 \mathrm{C}$ & 109.5 \\
\hline $\mathrm{C} 34-\mathrm{C} 27-\mathrm{C} 28$ & $108.39(16)$ & $\mathrm{H} 35 \mathrm{~A}-\mathrm{C} 35-\mathrm{H} 35 \mathrm{C}$ & 109.5 \\
\hline $\mathrm{C} 35-\mathrm{C} 27-\mathrm{C} 28$ & $110.15(18)$ & $\mathrm{H} 35 \mathrm{~B}-\mathrm{C} 35-\mathrm{H} 35 \mathrm{C}$ & 109.5 \\
\hline $\mathrm{C} 34-\mathrm{C} 27-\mathrm{C} 26$ & $109.22(16)$ & $\mathrm{C} 5-\mathrm{C} 12-\mathrm{H} 12 \mathrm{~A}$ & 109.5 \\
\hline $\mathrm{C} 35-\mathrm{C} 27-\mathrm{C} 26$ & $109.83(16)$ & $\mathrm{C} 5-\mathrm{C} 12-\mathrm{H} 12 \mathrm{~B}$ & 109.5 \\
\hline $\mathrm{C} 28-\mathrm{C} 27-\mathrm{C} 26$ & $107.59(15)$ & $\mathrm{H} 12 \mathrm{~A}-\mathrm{C} 12-\mathrm{H} 12 \mathrm{~B}$ & 109.5 \\
\hline $\mathrm{C} 27-\mathrm{C} 28-\mathrm{C} 29$ & $111.83(14)$ & $\mathrm{C} 5-\mathrm{C} 12-\mathrm{H} 12 \mathrm{C}$ & 109.5 \\
\hline $\mathrm{C} 27-\mathrm{C} 28-\mathrm{H} 28 \mathrm{~A}$ & 109.3 & $\mathrm{H} 12 \mathrm{~A}-\mathrm{C} 12-\mathrm{H} 12 \mathrm{C}$ & 109.5 \\
\hline $\mathrm{C} 29-\mathrm{C} 28-\mathrm{H} 28 \mathrm{~A}$ & 109.3 & $\mathrm{H} 12 \mathrm{~B}-\mathrm{C} 12-\mathrm{H} 12 \mathrm{C}$ & 109.5 \\
\hline $\mathrm{N} 2-\mathrm{C} 13-\mathrm{C} 21-\mathrm{C} 17$ & $-179.89(13)$ & $\mathrm{C} 35-\mathrm{C} 27-\mathrm{C} 26-\mathrm{C} 25$ & $-179.97(18)$ \\
\hline $\mathrm{C} 20-\mathrm{C} 13-\mathrm{C} 21-\mathrm{C} 17$ & $60.96(17)$ & $\mathrm{C} 28-\mathrm{C} 27-\mathrm{C} 26-\mathrm{C} 25$ & $-60.08(19)$ \\
\hline $\mathrm{C} 14-\mathrm{C} 13-\mathrm{C} 21-\mathrm{C} 17$ & $-60.81(17)$ & $\mathrm{C} 13-\mathrm{C} 14-\mathrm{C} 15-\mathrm{C} 16$ & $-58.79(19)$ \\
\hline $\mathrm{N} 1-\mathrm{C} 1-\mathrm{C} 2-\mathrm{C} 3$ & $179.87(13)$ & $\mathrm{C} 13-\mathrm{C} 14-\mathrm{C} 15-\mathrm{C} 22$ & $59.17(19)$ \\
\hline $\mathrm{C} 9-\mathrm{C} 1-\mathrm{C} 2-\mathrm{C} 3$ & $60.33(17)$ & $\mathrm{C} 13-\mathrm{C} 14-\mathrm{C} 15-\mathrm{C} 23$ & $-179.39(18)$ \\
\hline $\mathrm{C} 8-\mathrm{C} 1-\mathrm{C} 2-\mathrm{C} 3$ & $-61.22(17)$ & $\mathrm{C} 2-\mathrm{C} 3-\mathrm{C} 4-\mathrm{C} 5$ & $59.02(19)$ \\
\hline $\mathrm{N} 1-\mathrm{C} 1-\mathrm{C} 8-\mathrm{C} 7$ & $-179.81(14)$ & $\mathrm{C} 11-\mathrm{C} 3-\mathrm{C} 4-\mathrm{C} 5$ & $-179.90(16)$ \\
\hline $\mathrm{C} 9-\mathrm{C} 1-\mathrm{C} 8-\mathrm{C} 7$ & $-60.78(18)$ & $\mathrm{C} 10-\mathrm{C} 3-\mathrm{C} 4-\mathrm{C} 5$ & $-58.78(19)$ \\
\hline $\mathrm{C} 2-\mathrm{C} 1-\mathrm{C} 8-\mathrm{C} 7$ & $60.66(18)$ & $\mathrm{C} 24-\mathrm{C} 17-\mathrm{C} 16-\mathrm{C} 15$ & $180.00(16)$ \\
\hline $\mathrm{N} 2-\mathrm{C} 13-\mathrm{C} 14-\mathrm{C} 15$ & $-179.95(14)$ & $\mathrm{C} 18-\mathrm{C} 17-\mathrm{C} 16-\mathrm{C} 15$ & $58.4(2)$ \\
\hline $\mathrm{C} 21-\mathrm{C} 13-\mathrm{C} 14-\mathrm{C} 15$ & $60.69(18)$ & $\mathrm{C} 21-\mathrm{C} 17-\mathrm{C} 16-\mathrm{C} 15$ & $-59.2(2)$ \\
\hline $\mathrm{C} 20-\mathrm{C} 13-\mathrm{C} 14-\mathrm{C} 15$ & $-60.94(18)$ & $\mathrm{C} 22-\mathrm{C} 15-\mathrm{C} 16-\mathrm{C} 17$ & $-58.15(19)$ \\
\hline $\mathrm{N} 3-\mathrm{C} 25-\mathrm{C} 33-\mathrm{C} 29$ & $179.97(13)$ & $\mathrm{C} 23-\mathrm{C} 15-\mathrm{C} 16-\mathrm{C} 17$ & $179.57(17)$ \\
\hline $\mathrm{C} 26-\mathrm{C} 25-\mathrm{C} 33-\mathrm{C} 29$ & $-60.58(17)$ & $\mathrm{C} 14-\mathrm{C} 15-\mathrm{C} 16-\mathrm{C} 17$ & $59.6(2)$ \\
\hline $\mathrm{C} 32-\mathrm{C} 25-\mathrm{C} 33-\mathrm{C} 29$ & $61.26(17)$ & $\mathrm{C} 1-\mathrm{C} 9-\mathrm{C} 5-\mathrm{C} 12$ & $-179.83(19)$ \\
\hline $\mathrm{C} 1-\mathrm{C} 2-\mathrm{C} 3-\mathrm{C} 4$ & $-58.56(18)$ & $\mathrm{C} 1-\mathrm{C} 9-\mathrm{C} 5-\mathrm{C} 6$ & $-58.40(19)$ \\
\hline $\mathrm{C} 1-\mathrm{C} 2-\mathrm{C} 3-\mathrm{C} 11$ & $-179.59(14)$ & $\mathrm{C} 1-\mathrm{C} 9-\mathrm{C} 5-\mathrm{C} 4$ & $59.2(2)$ \\
\hline $\mathrm{C} 1-\mathrm{C} 2-\mathrm{C} 3-\mathrm{C} 10$ & $59.46(17)$ & $\mathrm{C} 3-\mathrm{C} 4-\mathrm{C} 5-\mathrm{C} 12$ & $-179.67(19)$ \\
\hline $\mathrm{N} 1-\mathrm{C} 1-\mathrm{C} 9-\mathrm{C} 5$ & $179.41(14)$ & $\mathrm{C} 3-\mathrm{C} 4-\mathrm{C} 5-\mathrm{C} 9$ & $-59.4(2)$ \\
\hline $\mathrm{C} 2-\mathrm{C} 1-\mathrm{C} 9-\mathrm{C} 5$ & $-60.65(18)$ & $\mathrm{C} 3-\mathrm{C} 4-\mathrm{C} 5-\mathrm{C} 6$ & $58.44(19)$ \\
\hline $\mathrm{C} 8-\mathrm{C} 1-\mathrm{C} 9-\mathrm{C} 5$ & $60.60(18)$ & $\mathrm{C} 25-\mathrm{C} 32-\mathrm{C} 31-\mathrm{C} 30$ & $59.1(2)$ \\
\hline $\mathrm{C} 25-\mathrm{C} 33-\mathrm{C} 29-\mathrm{C} 36$ & $178.98(17)$ & $\mathrm{C} 25-\mathrm{C} 32-\mathrm{C} 31-\mathrm{C} 34$ & $-60.8(2)$ \\
\hline $\mathrm{C} 25-\mathrm{C} 33-\mathrm{C} 29-\mathrm{C} 30$ & $-59.61(18)$ & $\mathrm{C} 30-\mathrm{C} 31-\mathrm{C} 34-\mathrm{C} 27$ & $-60.0(2)$ \\
\hline $\mathrm{C} 25-\mathrm{C} 33-\mathrm{C} 29-\mathrm{C} 28$ & $57.92(18)$ & $\mathrm{C} 32-\mathrm{C} 31-\mathrm{C} 34-\mathrm{C} 27$ & $60.4(2)$ \\
\hline $\mathrm{C} 13-\mathrm{C} 21-\mathrm{C} 17-\mathrm{C} 24$ & $-179.97(16)$ & $\mathrm{C} 35-\mathrm{C} 27-\mathrm{C} 34-\mathrm{C} 31$ & $179.94(18)$ \\
\hline
\end{tabular}


$\mathrm{C} 13-\mathrm{C} 21-\mathrm{C} 17-\mathrm{C} 16$
$\mathrm{C} 13-\mathrm{C} 21-\mathrm{C} 17-\mathrm{C} 18$
$\mathrm{~N} 2-\mathrm{C} 13-\mathrm{C} 20-\mathrm{C} 19$
$\mathrm{C} 21-\mathrm{C} 13-\mathrm{C} 20-\mathrm{C} 19$
$\mathrm{C} 14-\mathrm{C} 13-\mathrm{C} 20-\mathrm{C} 19$
$\mathrm{C} 1-\mathrm{C} 8-\mathrm{C} 7-\mathrm{C} 6$
$\mathrm{C} 1-\mathrm{C} 8-\mathrm{C} 7-\mathrm{C} 10$
$\mathrm{C} 6-\mathrm{C} 7-\mathrm{C} 10-\mathrm{C} 3$
$\mathrm{C} 8-\mathrm{C} 7-\mathrm{C} 10-\mathrm{C} 3$
$\mathrm{C} 2-\mathrm{C} 3-\mathrm{C} 10-\mathrm{C} 7$
$\mathrm{C} 4-\mathrm{C} 3-\mathrm{C} 10-\mathrm{C} 7$
$\mathrm{C} 11-\mathrm{C} 3-\mathrm{C} 10-\mathrm{C} 7$
$\mathrm{C} 34-\mathrm{C} 27-\mathrm{C} 28-\mathrm{C} 29$
$\mathrm{C} 35-\mathrm{C} 27-\mathrm{C} 28-\mathrm{C} 29$
$\mathrm{C} 26-\mathrm{C} 27-\mathrm{C} 28-\mathrm{C} 29$
$\mathrm{C} 36-\mathrm{C} 29-\mathrm{C} 28-\mathrm{C} 27$
$\mathrm{C} 30-\mathrm{C} 29-\mathrm{C} 28-\mathrm{C} 27$
$\mathrm{C} 33-\mathrm{C} 29-\mathrm{C} 28-\mathrm{C} 27$
N3-C25-C32-C 31
C33-C25-C32-C 31
C26-C25-C32-C 31
N3-C25-C26-C27
C33-C25-C26-C27
C32-C25-C26-C27
C34-C27-C26-C25
58.77 (18)

$-59.00(18)$

$-179.97(15)$

$-60.65(19)$

$61.13(19)$

60.18 (19)

$-60.2(2)$

$-60.18(19)$

$60.3(2)$

$-59.00(19)$

58.89 (19)

$180.00(16)$

$-58.18(19)$

$179.51(18)$

$59.8(2)$

$-179.52(17)$

$58.2(2)$

$-58.9(2)$

$-179.24(15)$

$-59.88(19)$

61.68 (19)

$-178.60(14)$

$61.69(18)$

$-59.91(18)$

57.37 (19)
$\mathrm{C} 28-\mathrm{C} 27-\mathrm{C} 34-\mathrm{C} 31$

$\mathrm{C} 26-\mathrm{C} 27-\mathrm{C} 34-\mathrm{C} 31$

C24-C17-C18-C19

$\mathrm{C} 16-\mathrm{C} 17-\mathrm{C} 18-\mathrm{C} 19$

$\mathrm{C} 21-\mathrm{C} 17-\mathrm{C} 18-\mathrm{C} 19$

$\mathrm{C} 34-\mathrm{C} 31-\mathrm{C} 30-\mathrm{C} 29$

$\mathrm{C} 32-\mathrm{C} 31-\mathrm{C} 30-\mathrm{C} 29$

$\mathrm{C} 36-\mathrm{C} 29-\mathrm{C} 30-\mathrm{C} 31$

$\mathrm{C} 28-\mathrm{C} 29-\mathrm{C} 30-\mathrm{C} 31$

$\mathrm{C} 33-\mathrm{C} 29-\mathrm{C} 30-\mathrm{C} 31$

$\mathrm{C} 16-\mathrm{C} 15-\mathrm{C} 22-\mathrm{C} 19$

$\mathrm{C} 23-\mathrm{C} 15-\mathrm{C} 22-\mathrm{C} 19$

$\mathrm{C} 14-\mathrm{C} 15-\mathrm{C} 22-\mathrm{C} 19$

$\mathrm{C} 8-\mathrm{C} 7-\mathrm{C} 6-\mathrm{C} 5$

$\mathrm{C} 10-\mathrm{C} 7-\mathrm{C} 6-\mathrm{C} 5$

$\mathrm{C} 12-\mathrm{C} 5-\mathrm{C} 6-\mathrm{C} 7$

$\mathrm{C} 9-\mathrm{C} 5-\mathrm{C} 6-\mathrm{C} 7$

$\mathrm{C} 4-\mathrm{C} 5-\mathrm{C} 6-\mathrm{C} 7$

$\mathrm{C} 17-\mathrm{C} 18-\mathrm{C} 19-\mathrm{C} 20$

$\mathrm{C} 17-\mathrm{C} 18-\mathrm{C} 19-\mathrm{C} 22$

$\mathrm{C} 13-\mathrm{C} 20-\mathrm{C} 19-\mathrm{C} 18$

$\mathrm{C} 13-\mathrm{C} 20-\mathrm{C} 19-\mathrm{C} 22$

$\mathrm{C} 15-\mathrm{C} 22-\mathrm{C} 19-\mathrm{C} 18$

$\mathrm{C} 15-\mathrm{C} 22-\mathrm{C} 19-\mathrm{C} 20$
$58.5(2)$

$-58.5(2)$

$179.54(17)$

$-58.6(2)$

$58.8(2)$

$59.6(2)$

$-59.9(2)$

$179.75(18)$

$-58.3(2)$

$59.08(19)$

$57.9(2)$

$179.75(18)$

$-59.8(2)$

$-60.2(2)$

60.35 (19)

$179.38(17)$

$58.7(2)$

$-58.82(19)$

$-60.1(2)$

$59.7(2)$

$60.0(2)$

$-60.3(2)$

$-59.5(2)$

60.8 (2)

Hydrogen-bond geometry $\left(\AA,{ }^{\circ}\right)$

\begin{tabular}{lllll}
\hline$D-\mathrm{H} \cdots A$ & $D-\mathrm{H}$ & $\mathrm{H} \cdots A$ & $D \cdots A$ & $D-\mathrm{H} \cdots A$ \\
\hline $\mathrm{N} 1-\mathrm{H} 1 A \cdots \mathrm{O} 7^{\mathrm{i}}$ & $0.90(2)$ & $1.92(2)$ & $2.819(2)$ & $177(2)$ \\
$\mathrm{N} 1-\mathrm{H} 1 B \cdots \mathrm{O} 1$ & $0.90(2)$ & $1.94(2)$ & $2.833(2)$ & $179(2)$ \\
$\mathrm{N} 1-\mathrm{H} 1 C \cdots \mathrm{O} 4$ & $0.89(2)$ & $1.96(2)$ & $2.844(2)$ & $170(2)$ \\
$\mathrm{N} 2-\mathrm{H} 2 A \cdots \mathrm{O} 2^{\mathrm{ii}}$ & $0.88(2)$ & $1.92(2)$ & $2.7991(18)$ & $179(2)$ \\
$\mathrm{N} 2-\mathrm{H} 2 B \cdots \mathrm{O} 9$ ii & $0.87(2)$ & $1.94(2)$ & $2.8090(19)$ & $175(2)$ \\
$\mathrm{N} 2-\mathrm{H} 2 C \cdots \mathrm{O} 6$ & $0.90(2)$ & $1.90(2)$ & $2.7923(19)$ & $177(2)$ \\
$\mathrm{N} 3-\mathrm{H} 3 A \cdots \mathrm{O} 3$ & $0.90(2)$ & $1.91(2)$ & $2.7717(19)$ & $159(2)$ \\
$\mathrm{N} 3-\mathrm{H} 3 B \cdots \mathrm{O} 5$ & $0.90(2)$ & $1.89(2)$ & $2.7752(19)$ & $172(2)$ \\
$\mathrm{N} 3-\mathrm{H} 3 C \cdots \mathrm{O} 8^{\mathrm{i}}$ & $0.89(2)$ & $1.90(2)$ & $2.785(2)$ & $172(2)$ \\
$\mathrm{C} 39-\mathrm{H} 39 B \cdots 6^{\mathrm{iii}}$ & 0.96 & 2.59 & $3.423(3)$ & 145 \\
\end{tabular}

Symmetry codes: (i) $x+1, y, z$; (ii) $x, y-1, z$; (iii) $-x+1,-y+1,-z+1$. 